\title{
The vendor location problem
}

\author{
Yüce Çınar, Hande Yaman* \\ Bilkent University, Department of Industrial Engineering, 06800 Ankara, Turkey
}

\section{A R T I C L E I N F O}

Available online 4 March 2011

Keywords:

Location

Vendor location problem

Hierarchical facility location

Valid inequalities

Computational complexity

\begin{abstract}
A B S T R A C T
The vendor location problem is the problem of locating a given number of vendors and determining the number of vehicles and the service zones necessary for each vendor to achieve at least a given profit. We consider two versions of the problem with different objectives: maximizing the total profit and maximizing the demand covered. The demand and profit generated by a demand point are functions of the distance to the vendor. We propose integer programming models for both versions of the vendor location problem. We then prove that both are strongly NP-hard and we derive several families of valid inequalities to strengthen our formulations. We report the outcomes of a computational study where we investigate the effect of valid inequalities in reducing the duality gaps and the solution times for the vendor location problem.
\end{abstract}

(c) 2011 Elsevier Ltd. All rights reserved.

\section{Introduction}

With a major beverage company about to launch its own brand for demijohn water, we recently worked on the following discrete facility location problem.

Unlike drinks sold in regular bottles, demijohn water has the distinctive feature of making it hard for customers to switch brands; every brand has its own containers and customers pay for the first container, replacing it when empty with a full one. In this way, the customer then continues to only pay for the contents of the bottles; switching brands would mean they would have to pay for a full bottle again. Suppliers of bottled gas for cooking and heating purposes also benefit from this quasimonopoly once the customer has made her choice of brands.

Water sold in large containers is the rule rather than the exception in Turkey: in 2008, $80 \%$ of consumption was demijohn water and the remaining $20 \%$ was water bottled in smaller containers. And the market itself is large: about 8.5 billion liters per year according to the Association of Packaged Water Producers in Turkey (SUDER [27]) and still expected to grow (by $10 \%$ in 2009).

A recent marketing survey carried out by the beverage company shows that customers value the quality of the water (taste, hygiene, chemical composition, etc.) and the quality of the service the most. The quality of the service is strongly related to service times and the satisfaction is affected by the presence of competitors in the same region who could provide shorter service times.

\footnotetext{
* Corresponding author. Tel.: +90 31229027 68; fax: +90312 2664054

E-mail addresses: yuce@bilkent.edu.tr (Y. Çınar), hyaman@bilkent.edu.tr (H. Yaman).
}

The number of potential customers in a given region mainly depends on the distance to the assigned vendor and on the proximity of competitors. This explains why selling in many locations could increase the market share. This strategy, however, has a price: some vendors may not reach a given profit. The beverage company wanted to ensure that each vendor would earn enough money and that the company would maximize its market share.

Inspired by this real-life problem, we define the vendor location problem (VLP) as follows. We are given a set of demand points corresponding to population zones and a set of possible locations for vendors. Each vendor can only use a given number of vehicles. We also know the (fixed) cost of a vendor office (rent, insurance, salaries of employees at office, etc.) at a given location as well as the cost (including the salary of the driver) and capacity of a vehicle.

For a given demand point, there is a set of eligible vendors. Each demand point has a potential demand. The market share that our company can have depends on the travel times of its vendors and the proximity of competitors. The profit (sales revenue minus the transportation cost) therefore depends on the vendor that serves a demand point.

The VLP is the problem of locating a given number of vendors and assigning each demand point to at most one vehicle of an eligible vendor such that capacities of vehicles are not exceeded and each vendor achieves at least a determined profit. We consider two objective functions. In ProfitVLP, the aim is to maximize the total profit and in CoverageVLP, the aim is to maximize the coverage, i.e., the total demand served.

Our problem can be seen as a hierarchical facility location problem where demand points are in level 0 , vehicles are level 1 facilities, and vendors are level 2 facilities. Sahin and Sural [28] 
review hierarchical facility location models and propose a classification scheme. The first attribute in this scheme is flow pattern. In a single flow pattern, the flow starts from level 0 and ends at the highest level by passing through all intermediate levels. In a multiple flow pattern, flows can travel from any lower level to any higher level. Our problem has a single flow pattern in the opposite direction. The second attribute is service varieties. Here in a nested system, a higher level facility provides all services provided by a lower level facility; in a non-nested system, facilities in different levels provide different services. Our system is a non-nested system. As the third attribute, the authors consider the spatial configuration. In a coherent system, all demand that is served by a given lower level facility is served by the same higher level facility. Since in our system, each vehicle belongs to a vendor, we have a coherent system. The final attribute is the objective. Here the authors consider the three common objectives: median, covering, and fixed charge. ProfitVLP can be considered as a median type problem even though we maximize profit rather than minimize cost. CoverageVLP is a maximum covering type problem.

Multi-level facility location problems have been previously studied by many researchers. Aardal et al. [2] propose some facet defining and valid inequalities for the polytope associated with the two level uncapacitated facility location problem. Approximation algorithms are studied by Aardal et al. [1], Ageev [3], Ageev et al. [4], Bumb [9], Bumb and Kern [10], Gabor and van Ommeren [14], Guha et al. [16], Meyerson et al. [22], Shmoys et al. [26], Zhang [32], and Zhang and Ye [33]. Branch and bound algorithms are given by Kaufman et al. [18], Ro and Tcha [25], Tcha and Lee [29], and Tragantalerngsak et al. [31]. Barros and Labbé [7] present various formulations, a Lagrangean relaxation, and a primal heuristic. Gao and Robinson [12,13] propose dualbased solution procedures. Chardaire et al. [11] present two formulations, valid inequalities, a Lagrangian relaxation, and a simulated annealing algorithm. Linear and Lagrangian relaxations are studied by Bloemhof-Ruwaard et al. [8], Marín [20], Marín and Pelegrín [21], Pirkul and Jayaraman [24], Tragantalerngsak et al. [30] for different versions of the problem.

A recent work that is closely related to ours is on the capacity and distance constrained plant location problem by AlbaredaSambola et al. [5]. In this problem, a set of possible locations is given. A facility may house a number of identical vehicles. Each demand point must be assigned to a single vehicle of a facility. There are capacity restrictions for facilities and restrictions on the total distance traveled for vehicles. The aim is to determine where to open facilities, to decide on the number of vehicles for each facility, and to assign the demand points to vehicles and facilities with the aim of minimizing the costs of opening facilities, using vehicles, and assigning demand points to facilities and vehicles. The authors provide different models and a tabu search algorithm for this problem. This study is similar to ours in that it is concerned with assigning demand points to facility vehicles. It is different from ours in that it has capacity constraints for facilities and restrictions on the total distance traveled for vehicles; we have capacity constraints for vehicles and minimum profit constraints for facilities.

In this paper, we introduce two new two-level facility location problems, namely ProfitVLP and CoverageVLP, which are motivated by a real life problem. Different from the classical facility location problems, here we have minimum profit constraints for open facilities and capacity constraints for their vehicles. We investigate the computational complexity of these problems and prove that they are strongly NP-hard. We propose integer programming formulations, valid inequalities, and extra constraints to be able to use the cutting planes of off-the-shelf integer programming solvers. We report the outcomes of a computational study where we use four types of instances that differ in their demand and profit functions. We investigate the effect of valid inequalities on linear programming relaxation bounds and solution times for these different types of instances. Finally, we analyze the optimal solutions of ProfitVLP and CoverageVLP and report how the differences in demand and profit functions effect the service regions for an example problem. Hence, the contributions of the paper are two new facility location problems motivated by a real life problem, resolution of the status of their computational complexity, and strong mixed integer programming formulations for these problems.

The paper is organized as follows. In Section 2, we present integer programming formulations for ProfitVLP and CoverageVLP and prove that both problems are strongly NP-hard. We propose some valid inequalities in Section 3. Computational results are given in Section 4. We analyze the solutions of ProfitVLP and CoverageVLP for two different types of instances in Section 5. In Section 6, we conclude the paper.

\section{Formulations and complexity}

In this section, we first introduce the notation and then present formulations for ProfitVLP and CoverageVLP. Then we prove that both ProfitVLP and CoverageVLP are strongly NP-hard.

Let $I$ be the set of demand points and $J$ be the set of possible locations for vendors. For a demand point $i \in I, J_{i}$ is the set of vendors that can serve $i$. In our problem, we define $J_{i}$ to be the set of vendors whose travel time to $i$ does not exceed a given bound. We also define $I_{j}=\left\{i \in I: j \in J_{i}\right\}$ for $j \in J$.

We denote with $f_{j}$ the fixed cost of the vendor office and with $v_{j}$ the fixed cost of a vehicle for a vendor located at $j \in J$. We assume that these cost values are non-negative. We define $\rho_{\min }$ to be the minimum profit a vendor should achieve.

We denote with $p$ the number of vendors to be located. The vendor at location $j \in J$ may have up to $k_{j}^{\max }$ vehicles. Let $K_{j}=\left\{1, \ldots, k_{j}^{\max }\right\}$ for $j \in J$. The capacity of a vehicle is equal to $\gamma$.

Demand point $i \in I$ has demand $q_{i j}$ and generates profit $\rho_{i j}$ if it is served by the vendor at location $j \in J_{i}$. We assume that $q_{i j}$ 's are positive and that $\rho_{i j}$ 's are non-negative.

We define the following decision variables. For $i \in I, j \in J_{i}$, and $k \in K_{j}, x_{i j k}$ is 1 if demand point $i$ is assigned to vehicle $k$ of vendor $j$ and 0 otherwise, for $j \in J$, and $k \in K_{j}, z_{j k}$ is 1 if vendor $j$ uses vehicle $k$ and 0 otherwise, and finally, for $j \in J, y_{j}$ is 1 if a vendor is located at location $j$ and 0 otherwise.

Using these variables, the ProfitVLP can be modeled as follows:

$$
\max \sum_{i \in I} \sum_{j \in J_{i}} \sum_{k \in K_{j}} \rho_{i j} x_{i j k}-\sum_{j \in J} \sum_{k \in K_{j}} v_{j} z_{j k}-\sum_{j \in J} f_{j} y_{j}
$$

s.t. $\sum_{j \in J_{i}} \sum_{k \in K_{j}} x_{i j k} \leq 1 \quad \forall i \in I$

$\sum_{j \in J} y_{j}=p$

$\sum_{k \in K_{j}} x_{i j k} \leq y_{j} \quad \forall i \in I, j \in J_{i}$

$\sum_{i \in I_{j}} q_{i j} x_{i j k} \leq \gamma z_{j k} \quad \forall j \in J, k \in K_{j}$

$\sum_{i \in I_{j}} \rho_{i j} \sum_{k \in K_{j}} x_{i j k} \geq \sum_{k \in K_{j}} v_{j} z_{j k}+\left(\rho_{\min }+f_{j}\right) y_{j} \quad \forall j \in J$

$x_{i j k} \in\{0,1\} \quad \forall i \in I, j \in J_{i}, k \in K_{j}$

$z_{j k} \in\{0,1\} \quad \forall j \in J, k \in K_{j}$ 
$y_{j} \in\{0,1\} \quad \forall j \in J$

Constraints (2) ensure that a demand point is assigned to at most one vehicle of one eligible vendor. Constraint (3) states that the number of vendors to be located is $p$. If a vendor is not located at a given location, then a demand point cannot be served by any of its vehicles due to constraints (4). Constraints (5) are capacity constraints for vehicles. At the same time, they ensure that demand points are not assigned to vehicles that are not in use. Constraints (6) ensure that each vendor makes a profit of at least $\rho_{\min }$ units. Constraints (7)-(9) state that the variables are binary. Objective function (1) is the total profit of all vendors.

Note here that constraints $z_{j k} \leq y_{j}$ for $j \in J$ and $k \in K_{j}$ are not included in the model. Let $j \in J$ and $k \in K_{j}$. If there exists $i \in I_{j}$ with $x_{i j k}=1$, then constraints (4) force $y_{j}$ to one and constraints (5) force $z_{j k}$ to one. On the other hand, if $x_{i j k}=0$ for all $i \in I_{j}$, then there exists an optimal solution with $z_{j k}=0$ since $v_{j}$ 's are non-negative. Hence constraints $z_{j k} \leq y_{j}$ for $j \in J$ and $k \in K_{j}$ are not necessary for the validity of the model. We do not include them in the model not to increase the number of constraints. Later, we use them as valid inequalities and test their performance.

The CoverageVLP can be modeled as follows:

$\max \sum_{i \in I} \sum_{j \in J_{i}} \sum_{k \in K_{j}} q_{i j} x_{i j k}$

s.t. $\quad(2)-(9)$

Here the objective function (10) is the total demand served.

To conclude this section, we investigate the computational complexity of problems ProfitVLP and CoverageVLP.

Theorem 1. ProfitVLP and CoverageVLP are strongly NP-hard.

Proof. We prove that the decision versions of ProfitVLP and CoverageVLP are NP-complete in the strong sense by a reduction from the decision version of the bin packing problem.

Given a finite set of items $U$, a size $s_{i} \in \mathbb{Z}_{+}$for each $i \in U$, a positive integer bin capacity $B$, and a positive integer $\kappa$, the decision version of the bin packing problem is defined as follows. Is there a partition of set $U$ into $U_{1}, \ldots, U_{\kappa}$ such that $\sum_{i \in U_{u}} s_{i} \leq B$ for all $u=1, \ldots, \kappa$ ? This problem is NP-complete in the strong sense (see problem [SR1] in Garey and Johnson [15]).

First note that when $v_{j}=f_{j}=0$ for all $j \in J$ and $\rho_{i j}=q_{i j}$ for all $i \in I$ and $j \in J_{i}$, problems ProfitVLP and CoverageVLP become the same problem. Hence in the remaining part of the proof, we only consider CoverageVLP with $v_{j}=f_{j}=0$ for all $j \in J$ and $\rho_{i j}=q_{i j}$ for all $i \in I$ and $j \in J_{i}$.

We define the decision version of CoverageVLP as follows. Given the parameters of the problem and a positive constant $\Phi$, does there exist a feasible solution with coverage at least $\Phi$ ? This problem is in NP.

Given an instance of the bin packing problem, let $J$ be a singleton, $I=I_{1}=U, \quad p=1, \quad v_{1}=0, f_{1}=0, \quad \rho_{\min }=0, \quad k_{1}^{\max }=\kappa$, $\rho_{i 1}=q_{i 1}=s_{i}$ for $i \in I, \gamma=B$, and $\Phi=\sum_{i \in I} q_{i 1}$. Now there exists a solution to the decision version of the bin packing problem if and only if there exists a solution to the decision version of CoverageVLP. Hence, the decision version of CoverageVLP is NP-complete in the strong sense.

\section{Valid inequalities}

In this section, we propose some valid inequalities for both versions of the VLP.

Let $F$ be the set of solutions that satisfy constraints (2)-(9). We use some substructures in the formulation to derive our valid inequalities. We also propose some redundant constraints to convert some structures in our problem into knapsack structures so that we can use the lifted cover inequalities of off-the-shelf integer programming solvers.

\subsection{Lower bounds on the number of vehicles}

Albareda-Sambola et al. [5] propose the optimality cuts $\sum_{k \in K_{j}} z_{j k} \geq y_{j}$ for $j \in J$. These inequalities imply that if a vendor is open then it should use at least one vehicle. In our problem, since we have minimum profit constraints, in some cases we can obtain tighter bounds on the number of vehicles to be used by a vendor. Note that the resulting inequalities are valid inequalities for our problem rather than optimality cuts.

For $j \in J$ and a positive integer $m$, consider the following problem:

$\delta_{j}(m)=\max \sum_{i \in I_{j}} \sum_{k=1}^{m} \rho_{i j} \alpha_{i k}-\sum_{k=1}^{m} v_{j} \beta_{k}-f_{j}$

s.t. $\sum_{k=1}^{m} \alpha_{i k} \leq 1 \quad \forall i \in I_{j}$

$\sum_{i \in I_{j}} q_{i j} \alpha_{i k} \leq \gamma \beta_{k} \quad \forall k=1, \ldots, m$

$\alpha_{i k} \in\{0,1\} \quad \forall i \in I_{j}, k=1, \ldots, m$

$\beta_{k} \in\{0,1\} \quad \forall k=1, \ldots, m$

Here, the variable $\beta_{k}$ takes value 1 if vehicle $k=1, \ldots, m$ is used and takes value 0 otherwise, and the variable $\alpha_{i k}$ takes value 1 if demand point $i \in I_{j}$ is assigned to vehicle $k=1, \ldots, m$ and takes value 0 otherwise. Constraints (12) ensure that each demand point is assigned to at most one vehicle and constraints (13) ensure that the sum of demands of demand points assigned to a given vehicle does not exceed the capacity of the vehicle if the vehicle is in use and no demand points are assigned to this vehicle if it is not in use. The objective function is equal to the sum of profits of demand points that are assigned to some vehicle minus the sum of costs of using vehicles and the vendor office $j$.

This problem hence maximizes the total profit for vendor $j$ if vendor $j$ can use at most $m$ vehicles. Let $m_{j}$ be the smallest integer with $\delta_{j}\left(m_{j}\right) \geq \rho_{\min }$. Then for vendor $j$ to achieve a minimum level of profit of $\rho_{\min }$ units, it should have at least $m_{j}$ vehicles. If $m_{j}$ is a positive integer less than or equal to $k_{j}^{\max }$, then the inequality $\sum_{k \in K_{j}} z_{j k} \geq m_{j} y_{j}$ is a valid inequality. If $m_{j}$ does not exist or if $m_{j}>k_{j}^{\max }$, then vendor $j$ cannot be profitable. Hence we can set $y_{j}=0$.

The above problem is a capacitated facility location problem with single sourcing, which is an NP-hard problem (see, e.g., Neebe and Rao [23], Barcelo and Casanovas [6], Klincewicz and Luss [19], and Holmberg et al. [17]). As a result, computing the $\delta_{j}(m)$ values may be quite time consuming, hence we propose a way of computing lower bounds on $m_{j}$ values.

Proposition 1. Let $j \in J$ and $\sigma_{j}=\max _{i \in I_{j}} \rho_{i j} / q_{i j}$. The inequality

$\sum_{k \in K_{j}} z_{j k} \geq\left\lceil\frac{\rho_{\min }+f_{j}}{\sigma_{j} \gamma-v_{j}}\right\rceil y_{j}$

is valid for $F$.

Proof. For $j \in J, \sigma_{j} q_{i j} \geq \rho_{i j}$ for all $i \in I_{j}$. Multiplying constraints (5) with $\sigma_{j}$ and summing over $k \in K_{j}$ yields $\sum_{i \in I_{j}} \sigma_{j} q_{i j} \sum_{k \in K_{j}} x_{i j k} \leq$ $\sigma_{j} \gamma \sum_{k \in K_{j}} z_{j k}$. Since $\sigma_{j} q_{i j} \geq \rho_{i j}$ for all $i \in I_{j}$, this implies $\sum_{i \in I_{j}} \rho_{i j}$ $\sum_{k \in K_{j}} x_{i j k} \leq \sigma_{j} \gamma \sum_{k \in K_{j}} z_{j k}$. Now combining this with constraint (6), 
we obtain

$\sigma_{j} \gamma \sum_{k \in K_{j}} z_{j k} \geq \sum_{i \in I_{j}} \rho_{i j} \sum_{k \in K_{j}} x_{i j k} \geq \sum_{k \in K_{j}} v_{j} z_{j k}+\left(\rho_{\min }+f_{j}\right) y_{j}$

which gives

$\left(\sigma_{j} \gamma-v_{j}\right) \sum_{k \in K_{j}} z_{j k} \geq\left(\rho_{\min }+f_{j}\right) y_{j}$

This implies that if $y_{j}=1$, i.e., if a vendor is located at location $j$, then $\sum_{k \in K_{j}} z_{j k} \geq\left(\rho_{\min }+f_{j}\right) /\left(\sigma_{j} \gamma-v_{j}\right)$. Since the left hand side is integer in a feasible solution, we can round up the right hand side.

If $y_{j}=0$, then (16) becomes redundant. Hence we can conclude that inequality (16) is valid for $F$.

For $j \in J, \sigma_{j}$ can be computed in $O\left(\left|I_{j}\right|\right)$ time.

\subsection{Cover inequalities for vehicle capacity constraints}

For $i \in I, j \in J_{i}$, and $k \in K_{j}$, inequality

$x_{i j k} \leq z_{j k}$

is a valid inequality for $F$. These inequalities are often dominated by cover inequalities that may be generated using the knapsack structure of the capacity constraints (5) for the vehicles. Cover inequalities that are valid for each of these knapsack constraints are also valid for $F$. Let $j \in J, k \in K_{j}$, and $C \subseteq I_{j}$ be such that $\sum_{i \in C} q_{i j}>\gamma$. Then the cover inequality $\sum_{i \in C} x_{i j k} \leq(|C|-1) z_{j k}$ is a valid inequality for $F$. These inequalities can be strengthened by lifting.

Most of the integer programming solvers recognize knapsack constraints and use lifted cover inequalities as cutting planes. So here we limit our attention to some lifted cover inequalities that are not many in number so that they can be added to the formulation before giving it to the solver.

For a given location $j \in J$, we first consider all demand points with demand larger than half of the capacity of a vehicle. Then we know that at most one of these points may be assigned to a given vehicle of vendor $j$. This leads to the following set of inequalities.

Proposition 2. For $j \in J$ and $k \in K_{j}$, the lifted cover inequality

$\sum_{i \in I_{j}: q_{i j}>\gamma / 2} x_{i j k} \leq z_{j k}$

is valid for $F$.

Next, we generate lifted cover inequalities for each demand point $i \in I_{j}$ with demand not more than half the capacity.

Proposition 3. Let $i \in I_{j}$ be such that $q_{i j} \leq \gamma / 2$. Define $C_{i j}=\left\{l \in I_{j}\right.$ : $\left.q_{i j}+q_{l j}>\gamma\right\}$. Then the lifted cover inequality

$x_{i j k}+\sum_{l \in C_{i j}} x_{l j k} \leq z_{j k}$

is valid for $F$.

Proof. If $x_{i j k}=1$, then as $q_{i j}+q_{l j}>\gamma$ for each $l \in C_{i j}$, none of these demand points can be served by the same vehicle. If $x_{i j k}=0$, then as $q_{l j}+q_{m j}>\gamma$ for $l$ and $m$ in $C_{i j}$, we know that $\sum_{l \in C_{i j}} x_{l j k} \leq z_{j k}$.

Notice that if $C_{i j}$ is empty, then inequality (19) reduces to (17).

\subsection{Cover inequalities for the minimum profit constraints}

Finally, we propose to model the minimum profit constraints in a different way so that we can use the lifted cover cuts of off-the-shelf solvers. To this end, we complement sums of assignment variables and rewrite the minimum profit constraints as 0-1 knapsack constraints as follows.
Let $j \in J$. For $i \in I_{j}$, define the variable $\bar{x}_{i j}=1-\sum_{k \in K_{j}} x_{i j k}$. Notice that $\bar{x}_{i j}$ is a $0-1$ variable. Now the minimum profit constraint (6) can be rewritten as

$\sum_{i \in I_{j}} \rho_{i j} \geq \sum_{i \in I_{j}} \rho_{i j} \bar{x}_{i j}+\sum_{k \in K_{j}} v_{j} z_{j k}+\left(\rho_{\min }+f_{j}\right) y_{j}$

which is a $0-1$ knapsack inequality.

Now based on this substructure, we can derive cover inequalities that are valid for $F$.

Proposition 4. Let $j \in J, S_{1} \subseteq I_{j}$, and $S_{2} \subseteq K_{j}$ with $\left|S_{2}\right| v_{j}+\left(\rho_{\min }+f_{j}\right)>$ $\sum_{i \in I_{j} \backslash S_{1}} \rho_{i j}$. The inequality

$\sum_{k \in S_{2}} z_{j k} \leq \sum_{i \in S_{1}} \sum_{k \in K_{j}} x_{i j k}+\left(\left|S_{2}\right|-1\right) y_{j}$

is valid for $F$.

Proof. Let $j \in J$. Consider the knapsack inequality (20). Suppose that $y_{j}=1$. Let $S_{1} \subseteq I_{j}$ and $S_{2} \subseteq K_{j}$. If $\sum_{i \in S_{1}} \rho_{i j}+\left|S_{2}\right| v_{j}+\left(\rho_{\min }+f_{j}\right)>$ $\sum_{i \in I_{j}} \rho_{i j}$, then the cover inequality $\sum_{i \in S_{1}} \bar{x}_{i j}+\sum_{k \in S_{2}} z_{j k} \leq\left|S_{1}\right|+$ $\left|S_{2}\right|-1$ is valid. We can rewrite this inequality as $\sum_{i \in S_{1}}(1-$ $\left.\sum_{k \in K_{i}} x_{i j k}\right)+\sum_{k \in S_{2}} z_{j k} \leq\left|S_{1}\right|+\left|S_{2}\right|-1$, which simplifies to $\sum_{k \in S_{2}}$ $z_{j k} \leq \sum_{i \in S_{1}} \sum_{k \in K_{j}} x_{i j k}+\left|S_{2}\right|-1$.

If $y_{j}=0$, then $x_{i j k}=0$ for all $i \in I_{j}$ and $k \in K_{j}$ and $z_{j k}=0$ for all $k \in K_{j}$. Hence inequality (21) is valid for $F$.

\section{Computational results}

In this section, we report the outcomes of our computational study. Here, we investigate for which sizes we can solve the formulations to optimality in reasonable times and the effect of valid inequalities on the quality of upper bounds of linear programming relaxations and the solution times.

\subsection{The data set and models}

We use the data from the demijohn water company. The data includes 84 demand points, their estimated demands, the distances, and cost parameters. The set of possible locations for the vendors is the same as the set of demand points. Moreover, there is the additional restriction that if a vendor is located at a given demand point, then the demand of this point should be served by itself. To handle this, we added the constraint

$\sum_{k \in K_{j}} x_{j j k}=y_{j} \quad \forall j \in J$

We can also use this information to break the symmetry. We impose that if a vendor is located at a demand point, then the point should use its vehicle indexed as its first vehicle by adding the constraints

$x_{j j 1}=z_{j 1} \quad \forall j \in J$

$x_{j j 1}=y_{j} \quad \forall j \in J$

Let $P M 0$ and $C M 0$ be the models obtained by adding the above constraints to ProfitVLP and CoverageVLP, respectively. Let PM1 and $C M 1$ be the models $P M 0$ and $C M 0$ strengthened with the valid inequalities (16), which provide lower bounds on the number of vehicles for each vendor.

The fact that if a vendor is located at a demand point, then the point should use its first vehicle can further be used to obtain stronger lifted cover inequalities for the first vehicles:

$\sum_{\left.i \in I_{j} \backslash j\right\}: q_{i j}+q_{i j}>\gamma} x_{i j 1}=0 \quad \forall j \in J$ 
$\sum_{i \in I_{j} \backslash\langle j\}: q_{i j}>\left(\gamma-q_{i j}\right) / 2} x_{i j 1} \leq z_{j 1} \quad \forall j \in J$

$x_{i j 1}+\sum_{\left.l \in I_{j} \backslash j\right\}: q_{i j}+q_{l j}>\gamma-q_{j j}} x_{l j 1} \leq z_{j 1} \quad \forall j \in J, \quad i \in I_{j} \backslash\{j\}: q_{i j} \leq \frac{\gamma-q_{j j}}{2}$

We add the above cover inequalities for the first vehicles and inequalities (18) and (19) for the remaining vehicles to models $P M 1$ and $C M 1$ and call the resulting models PM2 and $C M 2$, respectively.

We remove constraints (6) from models PM2 and CM2 and add the following variables and constraints to obtain models PM3 and $C M 3$ :

$\bar{x}_{i j}=1-\sum_{k \in K_{j}} x_{i j k} \quad \forall i \in I, j \in J_{i}$

$\sum_{i \in I_{j}} \rho_{i j} \bar{x}_{i j}+\sum_{k \in K_{j}} v_{j} z_{j k}+\left(\rho_{\min }+f_{j}\right) y_{j} \leq \sum_{i \in I_{j}} \rho_{i j} \quad \forall j \in J$

$\bar{x}_{i j} \in\{0,1\} \quad \forall i \in I, j \in J_{i}$.

The aim is to enable the solver to see the knapsack structure in the minimum profit constraints so that it can generate cover inequalities as discussed in Section 3.3.

We add the simple valid inequalities

$z_{j k} \leq y_{j} \quad \forall j \in J, k \in K_{j}$

to models PM3 and CM3 to obtain models PM4 and CM4.

Finally, analyzing the results of our computational study, we also decided to repeat our experiment with additional models for ProfitVLP and CoverageVLP. For ProfitVLP, we tested model PM5, which is obtained by removing the cover inequalities obtained using vehicle capacity constraints, i.e., inequalities (18), (19), (25)-(27), from model PM4. For CoverageVLP, model CM5 is obtained by adding only valid inequalities $z_{j k} \leq y_{j}$ for all $j \in J$ and $k \in K_{j}$ to model CMO.

In Tables 1 and 2, we give the constraints of the different models for ProfitVLP and CoverageVLP, respectively.

To evaluate the performances of the models defined above, we used the following test set. We let $p \in\{4,6,8\}, k_{j}^{\max }=k^{\max } \in$ $\{6,8,10\}$ for all $j \in J$, and $\rho_{\min } \in\{50,100,150\}$.

For each value of $p, k^{\max }$, and $\rho_{\min }$, we have four problems with different demand and profit structures. In A-type problems, we take $q_{i j}=q_{i}$ and $\rho_{i j}=\rho_{i}$ for all $j \in J_{i}$ and $i \in I$. So in A-type instances, the demand and profit are independent of the distance between the demand point and its vendor. In B-type problems, we take $q_{i j}=q_{i}$ and $\rho_{i j}=c_{i j} q_{i}$ for all $j \in J_{i}$ and $i \in I$ where $c_{i j}$ is the unit profit that vendor $j$ gains if it serves demand point $i$ and is a function of the distance between $i$ and $j$. In C-type problems, we take $q_{i j}$ to be a function of the distance between $i$ and $j$ and $\rho_{i j}=c q_{i j}$ for all $j \in J_{i}$ and $i \in I$ where $c$ is the unit profit and does not depend on distances. In this case, we let $q_{i j}=q_{i}$ for vendors $j$ that are within a short traveling time of $i$ and then let $q_{i j}$ decrease with the distance between $i$ and $j$ for other eligible vendors. Precisely, for $i \in I$, we let $J_{i}=\left\{j \in J: d_{i j} \leq 10\right\}$, where $d_{i j}$ is the distance between the demand point $i$ and the vendor $j$. For $i \in I$ and $j \in J_{i}$, we let $q_{i j}=q_{i} \min \left\{1,\left(1.5-0.1 d_{i j}\right)\right\}$. Hence the demand generated by point $i$ is equal to $q_{i}$ if the vendor $j$ is within $5 \mathrm{~km}$ of point $i$ and is equal to $q_{i}\left(1.5-0.1 d_{i j}\right)$ if $j$ is farther. Finally, in $\mathbf{D}$-type problems, we take both the demands and the profits as functions of the distances.

Both problems ProfitVLP and CoverageVLP are infeasible for $\rho=150, p=8$, and all four demand and profit structures. These instances are removed from the results.

All models are solved using GAMS 22.5 and CPLEX 11.0.0 on an AMD Opteron 252 processor $(2.6 \mathrm{GHz}$ ) with $2 \mathrm{~GB}$ of RAM operating under the system CentOS (Linux version 2.6.9-42.0.3.ELsmp). We have a time limit of $1 \mathrm{~h}$.

\subsection{Results for ProfitVLP}

In Tables 3-6, we report the results for ProfitVLP and the four types of instances, $\mathbf{A}, \mathbf{B}, \mathbf{C}$, and $\mathbf{D}$, respectively. For each instance and model, we report the percentage gap between the upper bound obtained by solving the linear programming relaxation of the corresponding model and the best lower bound for the integer problem in the column LP gap. Then we report the cpu times in seconds. If the problem is not solved to optimality in $1 \mathrm{~h}$, then we report the remaining percentage gap in parentheses. Finally, we report the number of nodes in the branch and cut tree for each model and instance. The best results are marked bold.

Each table has a summary, where we can see the averages of linear programming relaxation gaps, final optimality gaps, cpu times, number of nodes, the number of instances solved to optimality with each model, and the number of times each model was among the best for the considered criterion.

In these tables we observe that the initial model PMO has huge duality gaps and adding the valid inequalities (16), which impose

Table 1

Constraints of the models for ProfitVLP.

\begin{tabular}{|c|c|c|c|c|c|}
\hline PMO & PM1 & PM2 & PM3 & PM4 & PM5 \\
\hline$(2)-(9)$ & $(2)-(9)$ & $(2)-(9)$ & $(2)-(5),(7)-(9)$ & $(2)-(5),(7)-(9)$ & $(2)-(5),(7)-(9)$ \\
\hline \multirow[t]{5}{*}{$(22)-(24)$} & $(22)-(24)$ & $(22)-(24)$ & $(22)-(24)$ & $(22)-(24)$ & $(22)-(24)$ \\
\hline & $(16)$ & $(16)$ & $(16)$ & $(16)$ & $(16)$ \\
\hline & & (18), (19), (25)-(27) & (18), (19), (25)-(27) & (18), (19), (25)-(27) & \\
\hline & & & $(28)-(30)$ & $(28)-(30)$ & $(28)-(30)$ \\
\hline & & & & $(31)$ & $(31)$ \\
\hline
\end{tabular}

Table 2

Constraints of the models for CoverageVLP.

\begin{tabular}{|c|c|c|c|c|c|}
\hline CMO & CM1 & CM2 & CM3 & CM4 & CM5 \\
\hline$(2)-(9)$ & $(2)-(9)$ & $(2)-(9)$ & $(2)-(5),(7)-(9)$ & $(2)-(5),(7)-(9)$ & $(2)-(9)$ \\
\hline \multirow[t]{5}{*}{$(22)-(24)$} & $(22)-(24)$ & $(22)-(24)$ & $(22)-(24)$ & $(22)-(24)$ & $(22)-(24)$ \\
\hline & $(16)$ & (16) & (16) & $(16)$ & \\
\hline & & (18), (19), (25)-(27) & (18), (19), (25)-(27) & (18), (19), (25)-(27) & \\
\hline & & & $(28)-(30)$ & $(28)-(30)$ & \\
\hline & & & & (31) & $(31)$ \\
\hline
\end{tabular}


Table 3

Results for ProfitVLP and A-type instances.

\begin{tabular}{|c|c|c|c|c|c|c|c|c|c|c|c|c|c|c|c|c|c|c|c|c|}
\hline \multicolumn{3}{|c|}{ Parameters } & \multicolumn{6}{|c|}{ LP gap (\%) } & \multicolumn{6}{|c|}{ Cpu time (s)/optimality gap (\%) } & \multicolumn{6}{|c|}{ Number of nodes } \\
\hline$\rho_{\min }$ & $k^{\max }$ & $p$ & $P M 0$ & PM1 & PM2 & PM3 & PM4 & PM5 & PMO & $P M 1$ & PM2 & PM3 & PM4 & PM5 & PMO & PM1 & PM2 & PM3 & PM4 & PM5 \\
\hline 50 & 6 & 4 & 76.77 & 76.77 & 29.21 & 29.21 & 6.48 & 6.48 & 397.61 & 203.00 & 284.25 & 454.59 & 261.57 & 177.23 & 29387 & 13208 & 14983 & 28200 & 13612 & 8503 \\
\hline 50 & 8 & 4 & 48.05 & 48.05 & 8.56 & 8.56 & 5.89 & 25.82 & 411.81 & 127.10 & 562.07 & 2874.28 & 866.48 & 1002.29 & 20792 & 7760 & 26693 & 103257 & 23868 & 43420 \\
\hline 50 & 10 & 4 & 42.51 & 42.51 & 3.92 & 3.92 & 3.92 & 42.31 & 244.58 & 153.81 & 32.81 & 12.25 & 9.46 & 43.52 & 27254 & 17360 & 3119 & 248 & 85 & 780 \\
\hline 50 & 6 & 6 & 58.36 & 58.36 & 9.26 & 9.26 & 5.04 & 21.57 & 116.99 & 110.11 & 123.83 & 196.02 & 166.54 & 110.79 & 3847 & 2575 & 4269 & 4660 & 3846 & 2894 \\
\hline 50 & 8 & 6 & 50.91 & 50.91 & 4.80 & 4.80 & 4.15 & 50.91 & 1180.66 & 568.44 & 60.53 & 112.02 & 91.72 & 653.58 & 49637 & 20099 & 929 & 1621 & 1683 & 28500 \\
\hline 50 & 10 & 6 & 49.93 & 49.93 & 4.12 & 4.12 & 4.12 & 49.93 & 287.11 & 772.12 & 149.12 & 84.97 & 215.28 & 407.10 & 14542 & 25647 & 1890 & 1496 & 2853 & 10654 \\
\hline 50 & 6 & 8 & 55.24 & 55.24 & 2.01 & 2.01 & 1.91 & 55.24 & 128.86 & 143.02 & 105.59 & 161.81 & 130.32 & 285.60 & 2767 & 2380 & 1437 & 2094 & 1617 & 3476 \\
\hline 50 & 8 & 8 & 55.09 & 55.09 & 1.93 & 1.93 & 1.93 & 55.09 & 262.26 & 280.51 & 286.32 & 218.60 & 318.31 & 471.21 & 5330 & 3551 & 4569 & 2248 & 3228 & 6962 \\
\hline 50 & 10 & 8 & 55.09 & 55.09 & 1.93 & 1.93 & 1.93 & 55.09 & 2062.02 & 996.55 & 1013.52 & 871.82 & 838.39 & 1068.35 & 57867 & 20087 & 19222 & 17513 & 8925 & 21266 \\
\hline 100 & 6 & 4 & 76.77 & 76.77 & 29.21 & 29.21 & 6.48 & 6.48 & 99.38 & 224.41 & 214.90 & 226.33 & 174.20 & 161.32 & 6639 & 13122 & 10132 & 12692 & 9235 & 7816 \\
\hline 100 & 8 & 4 & 48.05 & 48.05 & 8.56 & 8.56 & 5.89 & 25.82 & 907.42 & 330.63 & 1712.14 & 718.57 & 630.69 & 859.88 & 58284 & 13090 & 96939 & 39225 & 26042 & 39542 \\
\hline 100 & 10 & 4 & 42.51 & 42.51 & 3.92 & 3.92 & 3.92 & 42.31 & 93.30 & 67.80 & 20.82 & 10.61 & 7.48 & 30.33 & 7063 & 3413 & 504 & 274 & 64 & 533 \\
\hline 100 & 6 & 6 & 58.36 & 58.36 & 9.26 & 9.26 & 5.04 & 21.57 & 85.56 & 168.24 & 108.66 & 113.17 & 248.37 & 121.41 & 1947 & 7306 & 3870 & 2791 & 5043 & 2483 \\
\hline 100 & 8 & 6 & 50.91 & 50.91 & 4.77 & 4.77 & 4.12 & 50.91 & 565.66 & 593.62 & 73.19 & 80.21 & 117.88 & 312.07 & 18574 & 24299 & 1034 & 1032 & 1598 & 12511 \\
\hline 100 & 10 & 6 & 49.93 & 49.93 & 4.09 & 4.09 & 4.09 & 49.93 & 326.92 & 292.73 & 176.99 & 100.61 & 139.74 & 477.56 & 9777 & 11121 & 4441 & 1315 & 1655 & 14364 \\
\hline 100 & 6 & 8 & 55.24 & 55.24 & 1.84 & 1.84 & 1.82 & 55.24 & 270.22 & 159.86 & 330.99 & 310.32 & 408.94 & 285.72 & 4837 & 2495 & 4595 & 3669 & 4189 & 2963 \\
\hline 100 & 8 & 8 & 55.24 & 55.24 & 1.84 & 1.84 & 1.84 & 55.24 & 3259.19 & 1315.94 & 608.58 & 460.38 & 467.88 & 472.78 & 66262 & 30370 & 10986 & 3431 & 4171 & 4406 \\
\hline 100 & 10 & 8 & 55.24 & 55.24 & 1.84 & 1.84 & 1.84 & 55.24 & $(0.05)$ & $(0.05)$ & $(0.05)$ & 741.72 & 897.38 & 742.00 & 54635 & 50175 & 47481 & 4803 & 5475 & 5494 \\
\hline 150 & 6 & 4 & 76.77 & 76.77 & 29.21 & 29.21 & 6.48 & 6.48 & 427.28 & 205.37 & 340.14 & 142.27 & 166.03 & 132.58 & 34745 & 10641 & 23289 & 6411 & 7191 & 5580 \\
\hline 150 & 8 & 4 & 48.05 & 48.05 & 8.56 & 8.56 & 5.89 & 25.82 & 282.47 & 883.12 & 976.47 & 961.01 & 164.50 & 398.14 & 20508 & 46832 & 54511 & 38617 & 5468 & 22293 \\
\hline 150 & 10 & 4 & 42.51 & 42.51 & 3.92 & 3.92 & 3.92 & 42.31 & 27.77 & 25.76 & 19.65 & 9.84 & 36.53 & 52.64 & 738 & 690 & 638 & 160 & 555 & 661 \\
\hline 150 & 6 & 6 & 61.23 & 61.23 & 11.02 & 11.02 & 6.85 & 23.77 & 242.70 & 256.54 & 141.90 & 97.67 & 125.62 & 120.08 & 11248 & 6592 & 4629 & 1580 & 1757 & 3557 \\
\hline 150 & 8 & 6 & 55.77 & 55.77 & 7.06 & 7.06 & 6.76 & 55.77 & 366.69 & 249.83 & 619.59 & 52.91 & 139.67 & 108.36 & 10633 & 11690 & 23780 & 652 & 666 & 198 \\
\hline 150 & 10 & 6 & 54.73 & 54.73 & 6.35 & 6.35 & 6.35 & 54.73 & 979.08 & 2620.63 & $(0.04)$ & 202.62 & 279.85 & 163.29 & 35910 & 64156 & 115684 & 877 & 928 & 981 \\
\hline \multicolumn{3}{|c|}{$\begin{array}{l}\text { Average } \\
\text { Avg. opt. gap (\%) }\end{array}$} & 55.14 & 55.14 & 8.22 & 8.22 & 4.45 & 38.92 & $\begin{array}{c}691.24 \\
(\mathbf{0 . 0 0})\end{array}$ & $\begin{array}{c}597.88 \\
(\mathbf{0 . 0 0})\end{array}$ & $\begin{array}{c}631.76 \\
(\mathbf{0 . 0 0})\end{array}$ & $\begin{array}{c}383.94 \\
(\mathbf{0 . 0 0})\end{array}$ & $\begin{array}{c}287.62 \\
(0.00)\end{array}$ & $\begin{array}{c}360.74 \\
(\mathbf{0 . 0 0})\end{array}$ & 23051 & 17027 & 19984 & 11604 & 5573 & 10411 \\
\hline \multicolumn{3}{|c|}{ \# of solved ins. $(/ 24)$} & & & & & & & 23 & 23 & 22 & 24 & 24 & 24 & & & & & & \\
\hline \multicolumn{3}{|c|}{$\#$ of best solutions (/24) } & & & 10 & 10 & 24 & 3 & 2 & 4 & 3 & 10 & 4 & 1 & 2 & 4 & 2 & 9 & 4 & 3 \\
\hline
\end{tabular}


Table 4

Results for ProfitVLP and B-type instances.

\begin{tabular}{|c|c|c|c|c|c|c|c|c|c|c|c|c|c|c|c|c|c|c|c|c|}
\hline \multicolumn{3}{|c|}{ Parameters } & \multicolumn{6}{|c|}{ LP gap (\%) } & \multicolumn{6}{|c|}{ Cpu time (s)/optimality gap (\%) } & \multicolumn{6}{|c|}{ Number of nodes } \\
\hline$\rho_{\min }$ & $k^{\max }$ & $p$ & PMO & $P M 1$ & $P M 2$ & PM3 & PM4 & PM5 & PMO & PM1 & PM2 & PM3 & PM4 & PM5 & PMO & PM1 & PM2 & PM3 & PM4 & PM5 \\
\hline 50 & 6 & 4 & 77.61 & 77.61 & 29.38 & 29.38 & 6.93 & 7.78 & 118.79 & 243.27 & 403.13 & 435.34 & 394.87 & 157.66 & 6510 & 14967 & 26960 & 26043 & 23355 & 6967 \\
\hline 50 & 8 & 4 & 48.76 & 48.76 & 8.72 & 8.72 & 6.04 & 26.82 & 1004.05 & 2726.00 & 858.60 & 664.81 & 140.27 & 483.59 & 73179 & 103608 & 66030 & 33633 & 3897 & 16495 \\
\hline 50 & 10 & 4 & 43.51 & 43.51 & 4.32 & 4.32 & 4.32 & 43.31 & 80.74 & 185.76 & 10.28 & 19.22 & 20.70 & 54.16 & 4358 & 10526 & 278 & 396 & 368 & 690 \\
\hline 50 & 6 & 6 & 59.28 & 59.28 & 9.42 & 9.42 & 5.33 & 22.89 & 378.75 & 173.11 & 108.48 & 302.37 & 206.38 & 204.76 & 17782 & 8094 & 4562 & 7770 & 5924 & 10605 \\
\hline 50 & 8 & 6 & 52.04 & 52.04 & 5.12 & 5.12 & 4.55 & 52.02 & 368.67 & 119.70 & 149.56 & 71.49 & 147.88 & 165.26 & 14233 & 4161 & 5163 & 1678 & 2169 & 5203 \\
\hline 50 & 10 & 6 & 51.17 & 51.17 & 4.53 & 4.53 & 4.53 & 51.17 & 1969.78 & 350.27 & 118.53 & 144.66 & 203.60 & 1920.13 & 82051 & 13989 & 2111 & 2356 & 4122 & 51737 \\
\hline 50 & 6 & 8 & 57.31 & 57.31 & 2.38 & 2.38 & 2.21 & 57.23 & 277.26 & 308.33 & 101.84 & 172.87 & 178.26 & 213.94 & 7455 & 5374 & 1530 & 2400 & 1923 & 2837 \\
\hline 50 & 8 & 8 & 56.75 & 56.75 & 2.03 & 2.03 & 2.02 & 56.74 & 245.46 & 285.58 & 147.91 & 314.23 & 417.90 & 306.81 & 4151 & 4682 & 2970 & 4010 & 4293 & 4268 \\
\hline 50 & 10 & 8 & 56.75 & 56.75 & 2.04 & 2.04 & 2.04 & 56.75 & 765.83 & 680.23 & 428.27 & 563.48 & 1128.88 & 847.33 & 16686 & 13179 & 5266 & 6304 & 9672 & 9794 \\
\hline 100 & 6 & 4 & 77.61 & 77.61 & 29.38 & 29.38 & 6.93 & 7.78 & 625.21 & 392.02 & 168.70 & 187.98 & 204.20 & 698.42 & 49140 & 29850 & 10168 & 8666 & 14426 & 6520 \\
\hline 100 & 8 & 4 & 48.76 & 48.76 & 8.72 & 8.72 & 6.04 & 26.82 & 371.98 & 836.81 & 356.90 & 782.17 & 568.03 & 340.19 & 25341 & 37881 & 18964 & 34894 & 22507 & 14522 \\
\hline 100 & 10 & 4 & 43.51 & 43.51 & 4.32 & 4.32 & 4.32 & 43.31 & 148.09 & 44.02 & 8.64 & 17.50 & 39.84 & 68.94 & 10875 & 1288 & 307 & 348 & 576 & 1074 \\
\hline 100 & 6 & 6 & 59.28 & 59.28 & 9.41 & 9.41 & 5.33 & 22.89 & 201.71 & 256.55 & 218.15 & 239.38 & 428.11 & 180.65 & 10180 & 10857 & 8823 & 9104 & 18007 & 6085 \\
\hline 100 & 8 & 6 & 52.10 & 52.10 & 5.11 & 5.11 & 4.53 & 52.08 & 626.96 & 380.98 & 398.19 & 75.75 & 167.60 & 246.32 & 49338 & 28355 & 40180 & 1286 & 3042 & 10095 \\
\hline 100 & 10 & 6 & 51.23 & 51.23 & 4.52 & 4.52 & 4.52 & 51.23 & 539.51 & 338.08 & 222.25 & 148.77 & 189.52 & 755.32 & 28173 & 22321 & 9924 & 2428 & 3587 & 25125 \\
\hline 100 & 6 & 8 & 58.33 & 58.32 & 2.81 & 2.81 & 2.70 & 58.25 & 861.99 & 1562.33 & 1284.50 & 661.40 & 506.51 & 447.86 & 30967 & 35979 & 24283 & 6785 & 4620 & 6645 \\
\hline 100 & 8 & 8 & 57.53 & 57.53 & 2.29 & 2.29 & 2.27 & 57.53 & $(0.21)$ & $(0.03)$ & 2493.71 & 743.06 & 775.63 & 1250.77 & 80039 & 112763 & 56433 & 7183 & 7549 & 14744 \\
\hline 100 & 10 & 8 & 57.31 & 57.31 & 2.15 & 2.15 & 2.15 & 57.31 & $(0.06)$ & $(0.01)$ & $(0.05)$ & 1384.88 & 1296.78 & 1069.77 & 70320 & 87082 & 101959 & 9137 & 9011 & 11935 \\
\hline 150 & 6 & 4 & 77.61 & 77.61 & 29.38 & 29.38 & 6.93 & 7.78 & 1263.19 & 107.18 & 154.69 & 217.05 & 214.49 & 156.04 & 76293 & 6924 & 7789 & 11128 & 12527 & 6577 \\
\hline 150 & 8 & 4 & 48.76 & 48.76 & 8.72 & 8.72 & 6.04 & 26.82 & 1382.57 & 2057.22 & 869.85 & 508.54 & 795.61 & 407.60 & 86130 & 120951 & 42405 & 31613 & 32705 & 19499 \\
\hline 150 & 10 & 4 & 43.51 & 43.51 & 4.32 & 4.32 & 4.32 & 43.31 & 147.35 & 32.47 & 22.35 & 11.43 & 18.68 & 77.87 & 13428 & 550 & 685 & 214 & 339 & 1622 \\
\hline 150 & 6 & 6 & 61.61 & 61.61 & 10.30 & 10.30 & 6.71 & 24.69 & 544.47 & 132.41 & 117.53 & 120.22 & 167.07 & 94.94 & 19639 & 4502 & 3275 & 2975 & 4778 & 3757 \\
\hline 150 & 8 & 6 & 56.82 & 56.82 & 6.78 & 6.78 & 6.50 & 56.80 & $(0.01)$ & 125.11 & 1006.17 & 88.80 & 124.49 & 152.24 & 113245 & 901 & 53107 & 664 & 660 & 1605 \\
\hline 150 & 10 & 6 & 55.87 & 55.87 & 6.15 & 6.15 & 6.15 & 55.87 & 111.98 & $(0.00)$ & 167.07 & 138.64 & 53.78 & 137.25 & 2345 & 89894 & 3173 & 743 & 573 & 764 \\
\hline \multicolumn{3}{|c|}{ Average } & 56.38 & 56.38 & 8.43 & 8.43 & 4.73 & 40.30 & $\begin{array}{r}951.44 \\
(0.01)\end{array}$ & $\begin{array}{r}922.41 \\
(\mathbf{0 . 0 0})\end{array}$ & $\begin{array}{l}558.98 \\
(\mathbf{0 . 0 0})\end{array}$ & $\begin{array}{c}333.91 \\
(0.00)\end{array}$ & $\begin{array}{r}349.55 \\
(\mathbf{0 . 0 0})\end{array}$ & $\begin{array}{c}434.91 \\
(\mathbf{0 . 0 0})\end{array}$ & 37161 & 32028 & 20681 & 8823 & 7943 & 9965 \\
\hline \multicolumn{3}{|c|}{ \# of solved ins. (/24) } & & & & & & & 21 & 21 & 23 & 24 & 24 & 24 & & & & & & \\
\hline \multicolumn{3}{|c|}{ \# of best solutions (/24) } & & & 8 & 8 & 24 & & 1 & 1 & 9 & 6 & 2 & 5 & 1 & & 7 & 6 & 5 & 5 \\
\hline
\end{tabular}


Table 5

Results for ProfitVLP and C-type instances.

\begin{tabular}{|c|c|c|c|c|c|c|c|c|c|c|c|c|c|c|c|c|c|c|c|c|}
\hline \multicolumn{3}{|c|}{ Parameters } & \multicolumn{6}{|c|}{ LP gap (\%) } & \multicolumn{6}{|c|}{ Cpu time (s)/optimality gap (\%) } & \multicolumn{6}{|c|}{ Number of nodes } \\
\hline$\rho_{\min }$ & $k^{\max }$ & $p$ & PMO & $P M 1$ & PM2 & PM3 & PM4 & PM5 & PMO & $P M 1$ & PM2 & PM3 & PM4 & PM5 & PMO & $P M 1$ & $P M 2$ & PM3 & PM4 & PM5 \\
\hline 50 & 6 & 4 & 36.40 & 36.40 & 34.53 & 34.53 & 3.99 & 3.99 & 415.66 & 545.82 & 908.56 & 808.90 & 235.64 & 137.35 & 21173 & 29775 & 25878 & 16029 & 4582 & 5389 \\
\hline 50 & 8 & 4 & 13.23 & 13.23 & 11.75 & 11.75 & 11.27 & 12.69 & $(0.20)$ & $(0.77)$ & $(1.46)$ & 1823.53 & $(1.39)$ & $(0.39)$ & 263317 & 210493 & 33642 & 55561 & 113821 & 195620 \\
\hline 50 & 10 & 4 & 13.27 & 13.27 & 11.80 & 11.80 & 11.80 & 13.27 & (1.31) & $(2.43)$ & (2.19) & $(3.42)$ & (1.38) & (1.16) & 153821 & 125472 & 46926 & 64855 & 58345 & 115305 \\
\hline 50 & 6 & 6 & 21.78 & 21.78 & 17.42 & 17.42 & 14.10 & 14.80 & $(0.06)$ & $(0.02)$ & $(1.82)$ & $(0.23)$ & 2466.03 & $(0.35)$ & 89914 & 81063 & 22019 & 25754 & 25452 & 66734 \\
\hline 50 & 8 & 6 & 12.88 & 12.88 & 9.04 & 9.04 & 9.04 & 12.88 & 1029.34 & $(0.86)$ & (1.15) & $(0.16)$ & $(0.02)$ & (0.68) & 33616 & 52149 & 25086 & 65312 & 41496 & 57322 \\
\hline 50 & 10 & 6 & 12.88 & 12.88 & 9.04 & 9.04 & 9.04 & 12.88 & $(0.88)$ & $(0.81)$ & $(0.71)$ & $(0.86)$ & $(0.81)$ & (0.58) & 60059 & 55828 & 24680 & 31578 & 22146 & 67819 \\
\hline 50 & 6 & 8 & 18.37 & 18.37 & 11.74 & 11.74 & 10.90 & 17.56 & (1.72) & (1.47) & (1.30) & $(4.04)$ & (5.18) & (0.68) & 29311 & 25123 & 18448 & 17679 & 24301 & 57322 \\
\hline 50 & 8 & 8 & 16.49 & 16.49 & 9.96 & 9.96 & 9.96 & 16.49 & $(0.63)$ & $(0.74)$ & (1.19) & (1.10) & (1.01) & (0.55) & 22195 & 21204 & 15233 & 14515 & 14275 & 21764 \\
\hline 50 & 10 & 8 & 16.53 & 16.53 & 10.01 & 10.01 & 10.01 & 16.53 & (1.05) & $(1.26)$ & $(1.74)$ & $(1.96)$ & $(1.76)$ & (1.18) & 21175 & 21012 & 12847 & 11431 & 13802 & 18843 \\
\hline 100 & 6 & 4 & 36.40 & 36.40 & 34.53 & 34.53 & 3.99 & 3.99 & 901.64 & 1212.46 & 690.56 & 1312.17 & 227.35 & 157.72 & 44662 & 52312 & 21072 & 23443 & 3208 & 5496 \\
\hline 100 & 8 & 4 & 13.23 & 13.23 & 11.75 & 11.75 & 11.27 & 12.69 & 3191.66 & $(0.81)$ & $(0.67)$ & $(0.75)$ & $(0.58)$ & $(0.39)$ & 119696 & 182370 & 183491 & 72687 & 67202 & 217651 \\
\hline 100 & 10 & 4 & 13.27 & 13.27 & 11.80 & 11.80 & 11.80 & 13.27 & (3.44) & (2.10) & $(2.07)$ & (2.41) & $(2.58)$ & (1.37) & 94767 & 153907 & 61780 & 52502 & 42564 & 116855 \\
\hline 100 & 6 & 6 & 21.78 & 21.78 & 17.41 & 17.41 & 14.10 & 14.80 & $(0.58)$ & $(0.02)$ & $(0.06)$ & $(0.41)$ & 2271.14 & 1773.18 & 111839 & 75878 & 32600 & 28226 & 27477 & 24158 \\
\hline 100 & 8 & 6 & 12.88 & 12.88 & 8.95 & 8.95 & 8.95 & 12.88 & $(0.74)$ & $(0.47)$ & $(0.55)$ & $(0.06)$ & $(0.52)$ & $(0.48)$ & 69973 & 73212 & 43094 & 68349 & 41428 & 85315 \\
\hline 100 & 10 & 6 & 12.88 & 12.88 & 8.95 & 8.95 & 8.95 & 12.88 & $(0.66)$ & (0.59) & $(0.96)$ & $(0.76)$ & $(0.78)$ & $(0.81)$ & 87953 & 57383 & 25728 & 26137 & 46347 & 49519 \\
\hline 100 & 6 & 8 & 20.07 & 20.07 & 13.44 & 13.44 & 12.61 & 19.38 & (1.68) & (1.32) & $(0.64)$ & (1.49) & $(2.55)$ & 1.97 & 29552 & 23039 & 17985 & 14123 & 18713 & 22312 \\
\hline 100 & 8 & 8 & 16.49 & 16.49 & 9.95 & 9.95 & 9.95 & 16.49 & $(0.53)$ & $(0.30)$ & (2.43) & $(0.81)$ & (1.58) & $(0.91)$ & 23977 & 27750 & 13911 & 11026 & 11332 & 15121 \\
\hline 100 & 10 & 8 & 16.53 & 16.53 & 10.00 & 10.00 & 10.00 & 16.53 & (2.09) & $(1.31)$ & $(1.82)$ & $(2.36)$ & (1.17) & (2.99) & 20528 & 18319 & 9930 & 11658 & 9690 & 17731 \\
\hline 150 & 6 & 4 & 36.40 & 36.40 & 34.53 & 34.53 & 3.99 & 3.99 & 350.66 & 1091.24 & 783.26 & 3397.84 & 213.28 & 126.14 & 17131 & 61934 & 24382 & 106412 & 3816 & 4747 \\
\hline 150 & 8 & 4 & 13.23 & 13.23 & 11.75 & 11.75 & 11.27 & 12.69 & 2749.68 & $(0.73)$ & $(0.95)$ & 3295.62 & 3170.46 & $(0.57)$ & 245267 & 114423 & 85248 & 91799 & 118125 & 189367 \\
\hline 150 & 10 & 4 & 13.27 & 13.27 & 11.80 & 11.80 & 11.80 & 13.27 & (1.16) & $(2.20)$ & $(2.20)$ & $(2.19)$ & $(1.25)$ & (1.16) & 149194 & 132028 & 52135 & 82438 & 50298 & 153399 \\
\hline 150 & 6 & 6 & 21.78 & 21.69 & 17.20 & 17.20 & 14.10 & 14.80 & 1725.05 & $(0.02)$ & 2271.70 & $(0.39)$ & $(0.52)$ & 1430.80 & 46143 & 67638 & 31197 & 26376 & 32231 & 17171 \\
\hline 150 & 8 & 6 & 14.34 & 14.34 & 10.18 & 10.18 & 10.18 & 14.34 & $(1.34)$ & (1.34) & (1.18) & (0.99) & (1.55) & (1.14) & 80373 & 82554 & 52963 & 44136 & 42435 & 77732 \\
\hline 150 & 10 & 6 & 14.34 & 14.34 & 10.18 & 10.18 & 10.18 & 14.34 & (1.86) & $(1.77)$ & $(1.77)$ & $(1.77)$ & (1.41) & (1.49) & 48787 & 58336 & 30143 & 34224 & 53502 & 67901 \\
\hline \multicolumn{3}{|c|}{$\begin{array}{l}\text { Average } \\
\text { Avg opt gap (\%) }\end{array}$} & 18.28 & 18.28 & 14.49 & 14.49 & 10.14 & 13.23 & $\begin{array}{r}\mathbf{2 9 8 2 . 9 9} \\
(0.83)\end{array}$ & $\begin{array}{r}3268.79 \\
(0.89)\end{array}$ & $\begin{array}{r}3194.00 \\
(1.49)\end{array}$ & $\begin{array}{r}3293.33 \\
(1.09)\end{array}$ & $\begin{array}{r}3057.75 \\
(1.09)\end{array}$ & $\begin{array}{c}3001.11 \\
(\mathbf{0 . 7 8})\end{array}$ & 78518 & 75133 & 37934 & 41510 & 36941 & 69608 \\
\hline \multicolumn{3}{|c|}{$\#$ of solved ins. $(/ 24)$} & & & & & & & 7 & 3 & 4 & 5 & 6 & 5 & & & & & & \\
\hline \multicolumn{3}{|c|}{$\#$ of best solutions (/24) } & & & 13 & 13 & 24 & 3 & 5 & 2 & 1 & 3 & 3 & 11 & & & 8 & 4 & 11 & 1 \\
\hline
\end{tabular}


Table 6

Results for ProfitVLP and D-type instances.

\begin{tabular}{|c|c|c|c|c|c|c|c|c|c|c|c|c|c|c|c|c|c|c|c|c|}
\hline \multicolumn{3}{|c|}{ Parameters } & \multicolumn{6}{|c|}{ LP gap (\%) } & \multicolumn{6}{|c|}{ Cpu time (s)/optimality gap (\%) } & \multicolumn{6}{|c|}{ Number of nodes } \\
\hline$\rho_{\min }$ & $k^{\max }$ & $p$ & PMO & PM1 & PM2 & PM3 & PM4 & PM5 & PMO & $P M 1$ & $P M 2$ & PM3 & PM4 & PM5 & PMO & PM1 & PM2 & PM3 & PM4 & PM5 \\
\hline 50 & 6 & 4 & 36.72 & 36.72 & 34.79 & 34.79 & 4.31 & 4.77 & 561.12 & 488.34 & 633.79 & 1956.61 & 232.02 & 160.37 & 28765 & 25766 & 15534 & 34652 & 5139 & 7266 \\
\hline 50 & 8 & 4 & 13.44 & 13.44 & 11.93 & 11.93 & 11.47 & 12.93 & 1164.72 & 455.10 & $(0.28)$ & $(1.16)$ & (1.09) & 2809.47 & 67315 & 28114 & 94733 & 100649 & 56289 & 170833 \\
\hline 50 & 10 & 4 & 13.48 & 13.48 & 11.98 & 11.98 & 11.98 & 13.48 & (3.12) & (2.15) & (2.67) & $(2.30)$ & (1.64) & $(2.26)$ & 100368 & 110347 & 68070 & 28127 & 66041 & 111921 \\
\hline 50 & 6 & 6 & 21.92 & 21.92 & 17.43 & 17.43 & 13.98 & 14.99 & $(0.01)$ & $(0.01)$ & $(0.60)$ & $(0.70)$ & 1757.17 & 2004.23 & 84878 & 98031 & 62948 & 22232 & 14977 & 26490 \\
\hline 50 & 8 & 6 & 13.27 & 13.27 & 9.32 & 9.32 & 9.32 & 13.27 & (1.58) & $(0.74)$ & (0.73) & $(0.54)$ & $(0.71)$ & $(0.09)$ & 81713 & 56679 & 29972 & 30236 & 45316 & 72111 \\
\hline 50 & 10 & 6 & 13.27 & 13.27 & 9.32 & 9.32 & 9.32 & 13.27 & (1.04) & $(0.75)$ & $(0.86)$ & $(0.79)$ & $(0.62)$ & (0.82) & 52052 & 47068 & 19260 & 18872 & 40327 & 57108 \\
\hline 50 & 6 & 8 & 20.76 & 20.76 & 13.77 & 13.77 & 12.87 & 19.87 & (3.12) & (2.14) & (2.17) & $(2.63)$ & (4.98) & (2.56) & 32632 & 29856 & 21022 & 16450 & 26431 & 29340 \\
\hline 50 & 8 & 8 & 17.01 & 17.01 & 10.25 & 10.25 & 10.25 & 17.01 & (1.10) & $(0.90)$ & (1.36) & $(1.71)$ & (1.25) & (1.53) & 29339 & 18631 & 13130 & 15488 & 12540 & 22387 \\
\hline 50 & 10 & 8 & 17.03 & 17.03 & 10.27 & 10.27 & 10.27 & 17.03 & (1.33) & (1.62) & (1.69) & $(1.61)$ & (2.39) & (3.12) & 23767 & 20408 & 11139 & 11722 & 13368 & 20784 \\
\hline 100 & 6 & 4 & 36.72 & 36.72 & 34.79 & 34.79 & 4.31 & 4.77 & 3386.70 & 507.64 & 1106.28 & 1203.65 & 698.44 & 164.79 & 121859 & 20229 & 30277 & 20402 & 13817 & 7294 \\
\hline 100 & 8 & 4 & 13.44 & 13.44 & 11.93 & 11.93 & 11.47 & 12.93 & 1712.80 & (1.62) & 3393.36 & $(0.21)$ & 3577.48 & 2669.71 & 143447 & 187751 & 115416 & 91689 & 133343 & 154873 \\
\hline 100 & 10 & 4 & 13.48 & 13.48 & 11.98 & 11.98 & 11.98 & 13.48 & (2.29) & (2.14) & (3.88) & (1.46) & $(2.42)$ & $(0.82)$ & 150628 & 127016 & 61970 & 70603 & 47248 & 164907 \\
\hline 100 & 6 & 6 & 21.92 & 21.92 & 17.42 & 17.42 & 13.98 & 14.99 & $(0.58)$ & $(0.01)$ & $(0.01)$ & $(0.58)$ & 1718.09 & 1618.76 & 90554 & 77487 & 56953 & 24518 & 14288 & 17998 \\
\hline 100 & 8 & 6 & 13.27 & 13.27 & 9.21 & 9.21 & 9.21 & 13.27 & $(0.71)$ & $(0.09)$ & $(0.32)$ & 3097.56 & $(0.91)$ & $(0.64)$ & 72710 & 67244 & 35361 & 40838 & 80211 & 71418 \\
\hline 100 & 10 & 6 & 13.27 & 13.27 & 9.21 & 9.21 & 9.21 & 13.27 & $(0.93)$ & (1.19) & (0.81) & $(0.73)$ & $(0.81)$ & (0.73) & 48044 & 44638 & 31362 & 31477 & 21707 & 52541 \\
\hline 100 & 6 & 8 & 20.60 & 20.60 & 13.74 & 13.74 & 12.86 & 19.86 & (1.25) & $(0.47)$ & $(0.04)$ & $(2.09)$ & $(2.89)$ & (1.35) & 28266 & 29342 & 28226 & 15084 & 18162 & 19170 \\
\hline 100 & 8 & 8 & 17.01 & 17.01 & 10.24 & 10.24 & 10.24 & 17.01 & $(0.60)$ & $(0.78)$ & $(0.76)$ & $(2.54)$ & (1.35) & (1.71) & 25717 & 19918 & 13032 & 10990 & 14204 & 20374 \\
\hline 100 & 10 & 8 & 17.01 & 17.01 & 10.24 & 10.24 & 10.24 & 17.01 & (1.10) & $(1.22)$ & $(1.29)$ & $(2.77)$ & $(3.31)$ & $(1.22)$ & 22651 & 17197 & 11800 & 10205 & 11563 & 18160 \\
\hline 150 & 6 & 4 & 36.72 & 36.72 & 34.79 & 34.79 & 4.31 & 4.77 & 419.46 & 290.98 & 801.11 & 1717.41 & 224.96 & 178.18 & 23992 & 14481 & 38333 & 27987 & 4510 & 7150 \\
\hline 150 & 8 & 4 & 13.44 & 13.44 & 11.93 & 11.93 & 11.47 & 12.93 & 2384.95 & $(0.62)$ & $(1.68)$ & 932.03 & $(0.53)$ & 1703.53 & 118142 & 116417 & 99826 & 32216 & 110576 & 113171 \\
\hline 150 & 10 & 4 & 13.48 & 13.48 & 11.98 & 11.98 & 11.98 & 13.48 & $(2.10)$ & $(2.74)$ & $(2.99)$ & $(\mathbf{1 . 8 1})$ & $(2.42)$ & $(2.40)$ & 118407 & 194520 & 49374 & 75712 & 64753 & 86667 \\
\hline 150 & 6 & 6 & 22.45 & 22.45 & 17.82 & 17.82 & 14.60 & 15.60 & $(0.72)$ & $(0.01)$ & $(0.00)$ & $(2.17)$ & 3056.64 & 1750.43 & 59335 & 83396 & 46790 & 19144 & 25628 & 22813 \\
\hline 150 & 8 & 6 & 14.78 & 14.78 & 10.49 & 10.49 & 10.49 & 14.78 & $(1.40)$ & (1.33) & (1.48) & (1.35) & $(0.83)$ & (1.41) & 80001 & 83067 & 53054 & 39108 & 43934 & 53446 \\
\hline 150 & 10 & 6 & 14.78 & 14.78 & 10.49 & 10.49 & 10.49 & 14.78 & $(1.86)$ & (1.61) & (1.45) & (1.44) & (1.53) & (1.71) & 48006 & 61615 & 24584 & 26854 & 36213 & 61914 \\
\hline \multirow{3}{*}{\multicolumn{3}{|c|}{$\begin{array}{l}\text { Average } \\
\text { Avg. opt. gap (\%) } \\
\text { \# of solved ins. (/24) } \\
\text { \# of best solutions (/ }\end{array}$}} & 18.72 & 18.72 & 14.81 & 14.81 & 10.44 & 13.73 & $\begin{array}{r}3101.29 \\
(1.04)\end{array}$ & $\begin{array}{r}3072.64 \\
(\mathbf{0 . 9 2})\end{array}$ & $\begin{array}{r}3247.35 \\
(1.05)\end{array}$ & $\begin{array}{r}3221.22 \\
(1.19)\end{array}$ & $\begin{array}{r}3019.44 \\
(1.24)\end{array}$ & $\begin{array}{r}\mathbf{2 7 9 4 . 2 1} \\
(0.93)\end{array}$ & 68858 & 65801 & 43007 & 33965 & 38358 & 57922 \\
\hline & & & & & & & & & 6 & 4 & 4 & 5 & 7 & 9 & & & & & & \\
\hline & & & & & 13 & 13 & 24 & & 4 & 3 & 1 & 5 & 4 & 8 & & 1 & 5 & 10 & 7 & 1 \\
\hline
\end{tabular}


lower bounds on the number of vehicles, has almost no impact on these gaps. The average gaps are 55.14\%, 56.38\%, $18.28 \%$, and $18.72 \%$ for A, B, C, and D instances, respectively. Here we remark that even though they are still very large, the instances of types $\mathbf{C}$ and $\mathbf{D}$ (instances where demands depend on the distances) have much smaller gaps compared to the instances of types $\mathbf{A}$ and $\mathbf{B}$. This may be due to the fact that capacity constraints for vehicles are tighter for instances of type $\mathbf{A}$ and $\mathbf{B}$. Indeed, the average gaps for the model with cover inequalities, $P M 2$, are $8.22 \%, 8.43 \%$, $14.49 \%$, and $14.81 \%$ for A, B, C, and D, respectively. Here we see that these inequalities have reduced the gaps considerably for Aand B-type instances whereas their effect was much smaller for $\mathbf{C}$ and D-type instances. After all the valid inequalities are added, with model PM4, the average gaps are $4.45 \%, 4.73 \%, 10.14 \%$, and $10.44 \%$ for A, B, C, and D, respectively. Here we see that the valid inequalities are more effective in improving the quality of linear programming upper bounds for A- and B-type instances.

Except for D-type instances, model PM4 gives the smallest number of nodes on the average. If we compare the average number of nodes for the original model PMO and the ones for model PM4, we observe that the reductions are $75.82 \%, 78.63 \%$, $52.95 \%$, and $44.29 \%$ for A, B, C, and D instances, respectively. We can conclude that our valid inequalities are more effective in reducing the size of the branch and cut tree for $\mathbf{A}$ - and B-type instances.

For A-type instances, models PM1 and PM2 could solve 23 instances, model PM3 22 instances, and models PM4, PM5, and PM6 24 instances to optimality in $1 \mathrm{~h}$. The remaining gaps are quite small for the unsolved instances. The best average cpu time is given by model PM4 and is 58.39\% less than the average cpu time of the original model PMO. Model PM4 has given the best cpu for only four instances, whereas model PM3 has given the best cpu for 10 instances out of 24 . This model has the best average cpu time for B-type instances. It is interesting to note that for these instances, model PM2 has given the best cpu time for nine instances and model PM3 has given the best cpu time for six instances. But one of the instances could not be solved to optimality with model PM2. Models PMO and PM1 could not solve three instances to optimality. The average cpu time of PM3 is $64.90 \%$ less than the average cpu time of PMO. For these instances, we see that both cover inequalities based on the vehicle capacities and the knapsack inequalities for minimum profit constraints are quite effective in reducing the cpu times on the average.

ProfitVLP is harder for instances of types $\mathbf{C}$ and $\mathbf{D}$, where the demands are functions of distances. Here our valid inequalities are not useful in reducing cpu times and final gaps for unsolved instances. We see that models PMO and PM5 are the best in terms of cpu times and the number of instances solved to optimality, for C and D instances, respectively. The largest final gap for $\mathbf{C}$ instances is $1.41 \%$, and for D instances it is $2.14 \%$.

\subsection{Results for CoverageVLP}

We report the results for CoverageVLP and the four types of instances, A, B, C, and D in Tables 7-10, respectively. CoverageVLP turned out to be easier to solve compared to ProfitVLP for our instances. First of all, the duality gaps were smaller for the original formulation. The average gaps are $16.62 \%, 16.71 \%$, $5.02 \%$, and $5.03 \%$ for A, B, C, and D instances, respectively. Again, the instances of types $\mathbf{C}$ and $\mathbf{D}$ have smaller gaps. Our valid inequalities reduced the average duality gaps to $1.22 \%, 1.26 \%$, $1.06 \%$, and $1.09 \%$ for A, B, C, and D instances, respectively. Even though it looks like the reduction in the duality gaps is mostly due to the use of valid inequalities (31), the differences between the average gaps of models CM4 and CM5 show that some of the remaining valid inequalities are also effective in strengthening the original model for $\mathbf{A}$ - and $\mathbf{B}$-type instances.

In terms of number of nodes, CM4, the model with all valid inequalities, has given the best average results, decreasing the number of nodes by $78.77 \%, 75.51 \%, 72.79 \%$, and $86.52 \%$ compared to $C M 0$ for $\mathbf{A}, \mathbf{B}, \mathbf{C}$, and $\mathbf{D}$ instances, respectively.

Only model CM4 could solve all 24 type $\mathbf{A}$ instances to optimality in $1 \mathrm{~h}$ of cpu time. Its average cpu is $78.14 \%$ less than the average cpu of the original model CMO. Similar results are obtained for B-type instances. For both types of instances, CM4 performs much better than all other models in terms of average cpu times.

All our models solve the 24 C-type instances to optimality within the time limit. Among these, CM5 has the best average cpu time. Our model with all valid inequalities has an average cpu time of $96.23 \mathrm{~s}$, whereas model CM5 has an average cpu time of $68.41 \mathrm{~s}$. Hence for these instances, we can conclude that even though the valid inequalities are effective in reducing the duality gaps and the sizes of the branch and cut trees, other than the simple inequalities $z_{j k} \leq y_{j}$ for all $j \in J$ and $k \in K_{j}$, they are not very useful in reducing the cpu times.

Finally, for D-type instances, the model CM4 gives the best average cpu time, which is $73.04 \%$ less than the average cpu time for the original model. It is interesting to note that for these instances, model CM5 could not solve two problems to optimality.

\subsection{Improvements in linear programming bounds}

Here, we report the percentage improvement in linear programming bounds obtained by adding families of valid inequalities. We first solve the linear programming relaxation of the model without any valid inequalities. Then we add each family of valid inequalities separately to the original model. We use the inequalities (16), which impose lower bounds on the number of vehicles, cover inequalities (18), (19), (25)-(27), and the simple valid inequalities (31). We compute the percentage improvements in the linear programming bounds. The averages are reported in Table 11 .

Here we observe that the inequalities (16), which impose lower bounds on the number of vehicles, do not improve the linear programming bounds. The cover inequalities result in significant improvements for ProfitVLP, especially for A- and B-type instances. However, they are not as useful for CoverageVLP. The valid inequalities (31) improve the linear programming bounds for all problems, more for A- and B-type instances and less for $\mathbf{C}$ - and $\mathbf{D}$-type instances.

\subsection{Comparison of profit and coverage values}

In Table 12, we report the best profit and coverage values for all ranges of parameters considered in our experiment. Here, we observe that for a given $\rho_{\min }$ value, best profit and coverage values are achieved with medium or large $k^{\max }$ and $p$ values. We depict the profit values for A-type instances in Fig. 1. Similar behavior is observed for the other types of instances.

For CoverageVLP, the best coverage values are achieved with $p=8$ and $k^{\max }=8,10$ for $\rho_{\min }=50,100$ and with $p=6$ and $k^{\max }=10$ for $\rho_{\min }=150$. Increasing $p$ and $k^{\max }$ has a significant effect on the best coverage values.

\section{Analysis of example optimal solutions}

In this section, we analyze the optimal solutions for problems ProfitVLP and CoverageVLP for an example instance with 
Table 7

Results for CoverageVLP and A-type instances.

\begin{tabular}{|c|c|c|c|c|c|c|c|c|c|c|c|c|c|c|c|c|c|c|c|c|}
\hline \multicolumn{3}{|c|}{ Parameters } & \multicolumn{6}{|c|}{ LP gap (\%) } & \multicolumn{6}{|c|}{ Cpu time (s)/optimality gap (\%) } & \multicolumn{6}{|c|}{ Number of nodes } \\
\hline$\rho_{\min }$ & $k^{\max }$ & $p$ & CMO & $C M 1$ & CM2 & CM3 & CM4 & CM5 & CMO & CM1 & CM2 & CM3 & CM4 & CM5 & CMO & CM1 & CM2 & CM3 & CM4 & CM5 \\
\hline 50 & 6 & 4 & 54.24 & 53.24 & 53.83 & 53.83 & 1.91 & 1.91 & 261.90 & 175.16 & 374.14 & 246.76 & 146.60 & 113.41 & 19701 & 13054 & 25542 & 11277 & 8610 & 6198 \\
\hline 50 & 8 & 4 & 23.05 & 23.05 & 22.71 & 22.71 & 1.58 & 7.26 & 255.51 & 811.10 & 801.58 & 260.04 & 51.14 & 203.96 & 17862 & 53197 & 71304 & 15729 & 2597 & 8852 \\
\hline 50 & 10 & 4 & 5.77 & 5.77 & 5.66 & 5.66 & 0.62 & 5.64 & 17.32 & 10.26 & 14.79 & 24.50 & 15.63 & 8.96 & 863 & 811 & 745 & 654 & 715 & 629 \\
\hline 50 & 6 & 6 & 30.68 & 30.68 & 30.34 & 30.34 & 1.31 & 5.78 & 85.34 & 61.67 & 89.38 & 88.92 & 79.11 & 53.68 & 4634 & 2807 & 3692 & 2097 & 1910 & 1710 \\
\hline 50 & 8 & 6 & 9.27 & 9.27 & 9.27 & 9.27 & 1.15 & 9.27 & 71.74 & 49.29 & 39.93 & 99.65 & 68.32 & 42.57 & 1907 & 843 & 685 & 1333 & 570 & 752 \\
\hline 50 & 10 & 6 & 0.00 & 0.00 & 0.00 & 0.00 & 0.00 & 0.00 & 6.21 & 3.54 & 1.74 & 2.69 & 1.71 & 0.61 & 211 & 20 & o & o & 0 & o \\
\hline 50 & 6 & 8 & 9.21 & 9.21 & 9.21 & 9.21 & 0.44 & 9.21 & 1329.72 & 1996.49 & $(0.29)$ & $(0.30)$ & 106.77 & 148.13 & 233085 & 32144 & 40052 & 31589 & 625 & 3298 \\
\hline 50 & 8 & 8 & 0.00 & 0.00 & 0.00 & 0.00 & 0.00 & 0.00 & 3.79 & 16.66 & 4.00 & 13.34 & 12.14 & 33.74 & o & 250 & o & 8 & 10 & 380 \\
\hline 50 & 10 & 8 & 0.00 & 0.00 & 0.00 & 0.00 & 0.00 & 0.00 & 5.16 & 13.68 & 5.78 & 6.94 & 5.64 & 3.02 & 80 & 150 & o & $\mathbf{0}$ & $\mathbf{0}$ & 134 \\
\hline 100 & 6 & 4 & 54.24 & 54.24 & 53.70 & 53.70 & 1.91 & 1.91 & 219.06 & 209.80 & 183.54 & 339.86 & 131.53 & 88.21 & 16737 & 15631 & 9254 & 13453 & 6647 & 2866 \\
\hline 100 & 8 & 4 & 23.05 & 23.05 & 22.69 & 22.69 & 1.58 & 7.26 & 571.61 & 696.36 & 1687.35 & 543.48 & 78.93 & 888.37 & 32944 & 29950 & 66906 & 35824 & 6046 & 49649 \\
\hline 100 & 10 & 4 & 5.77 & 5.77 & 5.66 & 5.66 & 0.62 & 5.64 & 22.05 & 19.96 & 7.91 & 15.94 & 7.65 & 16.25 & 1326 & 945 & 257 & 690 & 544 & 1141 \\
\hline 100 & 6 & 6 & 30.68 & 30.68 & 30.34 & 30.34 & 1.31 & 5.78 & 44.16 & 63.87 & 75.78 & 112.94 & 97.16 & 52.00 & 1473 & 2951 & 2647 & 2710 & 2328 & 1069 \\
\hline 100 & 8 & 6 & 9.44 & 9.44 & 9.44 & 9.44 & 1.32 & 9.44 & 58.18 & 67.67 & 87.82 & 214.85 & 32.86 & 50.73 & 1426 & 2435 & 830 & 4594 & 425 & 1163 \\
\hline 100 & 10 & 6 & 0.00 & 0.00 & 0.00 & 0.00 & 0.00 & 0.00 & 8.88 & 4.77 & 2.19 & 3.38 & 1.66 & 1.28 & 381 & 67 & 0 & o & 0 & o \\
\hline 100 & 6 & 8 & 9.78 & 9.78 & 9.78 & 9.78 & 0.96 & 9.78 & 3412.02 & $(0.63)$ & $(0.57)$ & $(0.57)$ & 952.09 & 3014.50 & 29703 & 30739 & 30454 & 27678 & 8222 & 35885 \\
\hline 100 & 8 & 8 & 0.00 & 0.00 & 0.00 & 0.00 & 0.00 & 0.00 & $(0.88)$ & 220.31 & 25.54 & 142.29 & 199.87 & $(0.33)$ & 12777 & 1615 & 40 & 557 & 800 & 14182 \\
\hline 100 & 10 & 8 & 0.00 & 0.00 & 0.00 & 0.00 & 0.00 & 0.00 & 131.76 & 625.47 & 73.83 & 20.53 & 22.89 & 202.88 & 1516 & 7030 & 519 & 103 & 30 & 2267 \\
\hline 150 & 6 & 4 & 54.24 & 54.24 & 53.62 & 53.62 & 1.91 & 1.91 & 105.72 & 189.55 & 262.52 & 242.02 & 137.16 & 92.46 & 8900 & 15466 & 18429 & 8928 & 6324 & 5142 \\
\hline 150 & 8 & 4 & 23.05 & 23.05 & 22.69 & 22.69 & 1.58 & 7.26 & 947.77 & 88.44 & 238.58 & 1361.99 & 113.91 & 235.52 & 44811 & 5313 & 11125 & 40893 & 6276 & 9365 \\
\hline 150 & 10 & 4 & 5.77 & 5.77 & 5.66 & 5.66 & 0.62 & 5.64 & 19.76 & 23.46 & 20.99 & 8.33 & 13.13 & 13.19 & 1085 & 1275 & 968 & 576 & 611 & 800 \\
\hline 150 & 6 & 6 & 31.32 & 31.32 & 29.10 & 29.10 & 1.78 & 6.30 & 387.94 & 96.44 & 108.43 & 87.50 & 130.49 & 146.91 & 21164 & 2363 & 3592 & 1955 & 2730 & 1854 \\
\hline 150 & 8 & 6 & 12.38 & 12.38 & 10.35 & 10.35 & 3.69 & 12.38 & 765.14 & $(0.34)$ & 759.93 & 198.72 & 171.76 & 505.64 & 24278 & 78776 & 18036 & 963 & 809 & 18144 \\
\hline 150 & 10 & 6 & 6.99 & 6.99 & 5.08 & 5.08 & 5.08 & 6.99 & 188.60 & 916.46 & 189.82 & 135.33 & 158.56 & 278.16 & 3086 & 18242 & 642 & 480 & 491 & 4892 \\
\hline \multicolumn{3}{|c|}{ Average } & 16.62 & 16.62 & 16.21 & 16.21 & 1.22 & 4.97 & $\begin{array}{c}521.64 \\
(0.04)\end{array}$ & $\begin{array}{c}565.15 \\
(0.04)\end{array}$ & $\begin{array}{c}510.66 \\
(0.04)\end{array}$ & $\begin{array}{c}473.76 \\
(0.04)\end{array}$ & $\begin{array}{r}114.03 \\
(0.00)\end{array}$ & $\begin{array}{c}408.10 \\
(0.01)\end{array}$ & 11248 & 13170 & 12738 & 8420 & 2388 & 7099 \\
\hline \multicolumn{3}{|c|}{$\begin{array}{l}\text { Avg. opt. gap (\%) } \\
\text { \# of solved ins. (/24) }\end{array}$} & & & & & & & 23 & 22 & 22 & 22 & 24 & 23 & & & & & & \\
\hline \multicolumn{3}{|c|}{ solutions (/24) } & 6 & 6 & 7 & 7 & 24 & 9 & 2 & 1 & 2 & 4 & 7 & 8 & 1 & 1 & 6 & 5 & 10 & 9 \\
\hline
\end{tabular}


Table 8

Results for CoverageVLP and B-type instances.

\begin{tabular}{|c|c|c|c|c|c|c|c|c|c|c|c|c|c|c|c|c|c|c|c|c|}
\hline \multicolumn{3}{|c|}{ Parameters } & \multicolumn{6}{|c|}{ LP gap (\%) } & \multicolumn{6}{|c|}{ Cpu time (s)/optimality gap (\%) } & \multicolumn{6}{|c|}{ Number of nodes } \\
\hline$\rho_{\min }$ & $k^{\max }$ & $p$ & CMO & CM1 & CM2 & CM3 & CM4 & CM5 & CMO & $C M 1$ & CM2 & CM3 & CM4 & CM5 & CMO & $C M 1$ & CM2 & CM3 & CM4 & CM5 \\
\hline 50 & 6 & 4 & 54.24 & 54.24 & 53.75 & 53.75 & 1.91 & 1.91 & 193.65 & 249.32 & 443.58 & 229.15 & 182.90 & 93.13 & 16136 & 19605 & 26851 & 9866 & 5938 & 5315 \\
\hline 50 & 8 & 4 & 23.05 & 23.05 & 22.69 & 22.69 & 1.58 & 7.26 & 217.40 & 250.88 & 1371.16 & 326.72 & 100.75 & 653.08 & 15880 & 24977 & 58418 & 19454 & 6358 & 28674 \\
\hline 50 & 10 & 4 & 5.77 & 5.77 & 5.66 & 5.66 & 0.62 & 5.64 & 18.51 & 16.73 & 14.12 & 8.75 & 19.83 & 5.49 & 1125 & 898 & 658 & 622 & 968 & 498 \\
\hline 50 & 6 & 6 & 30.68 & 30.68 & 30.34 & 30.34 & 1.31 & 5.78 & 85.60 & 70.00 & 108.38 & 91.01 & 85.15 & 92.55 & 5087 & 3068 & 4460 & 2661 & 2254 & 3648 \\
\hline 50 & 8 & 6 & 9.27 & 9.27 & 9.27 & 9.27 & 1.15 & 9.27 & 46.20 & 58.52 & 70.34 & 126.16 & 35.54 & 43.66 & 1618 & 962 & 666 & 920 & 410 & 733 \\
\hline 50 & 10 & 6 & 0.00 & 0.00 & 0.00 & 0.00 & 0.00 & 0.00 & 2.78 & 3.43 & 1.96 & 1.58 & 1.49 & 1.62 & 20 & 49 & $\mathbf{0}$ & $\mathbf{0}$ & $\mathbf{0}$ & $\mathbf{0}$ \\
\hline 50 & 6 & 8 & 9.21 & 9.21 & 9.21 & 9.21 & 0.44 & 9.21 & 993.46 & 2832.36 & $(0.31)$ & 997.20 & 106.86 & 85.75 & 13939 & 34035 & 38492 & 10581 & 974 & 874 \\
\hline 50 & 8 & 8 & 0.00 & 0.00 & 0.00 & 0.00 & 0.00 & 0.00 & 71.84 & 73.22 & 9.42 & 26.15 & 17.15 & 23.76 & 845 & 786 & 20 & 50 & 10 & 400 \\
\hline 50 & 10 & 8 & 0.00 & 0.00 & 0.00 & 0.00 & 0.00 & 0.00 & 10.22 & 6.66 & 4.26 & 5.42 & 5.18 & 6.82 & 220 & 59 & $\mathbf{0}$ & $\mathbf{0}$ & $\mathbf{0}$ & 190 \\
\hline 100 & 6 & 4 & 54.24 & 54.24 & 53.65 & 53.65 & 1.91 & 1.91 & 193.65 & 351.52 & 270.66 & 303.63 & 138.82 & 58.47 & 16136 & 24076 & 15971 & 10857 & 7955 & 2824 \\
\hline 100 & 8 & 4 & 23.05 & 23.05 & 22.69 & 22.69 & 1.58 & 7.26 & 217.40 & 2141.64 & 300.77 & 1272.10 & 77.90 & 446.48 & 15880 & 129486 & 23257 & 73610 & 5558 & 24236 \\
\hline 100 & 10 & 4 & 5.77 & 5.77 & 5.66 & 5.66 & 0.62 & 5.64 & 17.30 & 22.74 & 11.46 & 20.61 & 15.67 & 11.45 & 978 & 1065 & 714 & 653 & 968 & 902 \\
\hline 100 & 6 & 6 & 30.68 & 30.68 & 30.28 & 30.28 & 1.31 & 5.78 & 67.19 & 63.94 & 113.23 & 106.41 & 76.37 & 65.47 & 3114 & 2682 & 6621 & 2508 & 1761 & 2360 \\
\hline 100 & 8 & 6 & 9.44 & 9.44 & 9.44 & 9.44 & 1.32 & 9.44 & 108.21 & 79.64 & 145.11 & 173.98 & 52.91 & 100.91 & 3842 & 2313 & 1113 & 1836 & 521 & 4664 \\
\hline 100 & 10 & 6 & 0.00 & 0.00 & 0.00 & 0.00 & 0.00 & 0.00 & 11.27 & 14.70 & 2.25 & 2.51 & 1.48 & 5.31 & 418 & 492 & $\mathbf{0}$ & $\mathbf{0}$ & $\mathbf{0}$ & 89 \\
\hline 100 & 6 & 8 & 9.78 & 9.78 & 9.78 & 9.78 & 0.96 & 9.78 & $(0.44)$ & $(0.46)$ & $(0.47)$ & $(0.36)$ & 2115.30 & 2009.85 & 29528 & 29173 & 31932 & 21391 & 15394 & 18291 \\
\hline 100 & 8 & 8 & 0.00 & 0.00 & 0.00 & 0.00 & 0.00 & 0.00 & $(0.88)$ & 1016.32 & 2016.47 & 686.79 & 481.46 & 1789.27 & 20365 & 11097 & 6476 & 1574 & 1477 & 10274 \\
\hline 100 & 10 & 8 & 0.00 & 0.00 & 0.00 & 0.00 & 0.00 & 0.00 & 725.10 & 343.28 & 42.00 & 11.03 & 58.59 & $(1.14)$ & 7686 & 3190 & 100 & 20 & 19 & 15575 \\
\hline 150 & 6 & 4 & 54.24 & 54.24 & 53.62 & 53.62 & 1.91 & 1.91 & 113.95 & 233.72 & 227.44 & 259.32 & 95.73 & 73.11 & 7836 & 18333 & 12214 & 10286 & 3105 & 4149 \\
\hline 150 & 8 & 4 & 23.05 & 23.05 & 22.69 & 22.69 & 1.58 & 7.26 & 1028.98 & 598.60 & 160.18 & 232.00 & 71.69 & 18.17 & 68400 & 32616 & 8133 & 13838 & 4084 & 5525 \\
\hline 150 & 10 & 4 & 5.77 & 5.77 & 5.66 & 5.66 & 0.62 & 5.64 & 23.13 & 23.08 & 9.60 & 10.04 & 8.53 & 18.17 & 1200 & 1301 & 491 & 466 & 645 & 776 \\
\hline 150 & 6 & 6 & 31.32 & 31.32 & 28.23 & 28.23 & 1.76 & 6.30 & 100.34 & 52.67 & 162.01 & 62.76 & 64.42 & 96.12 & 7090 & 1542 & 7745 & 1524 & 1489 & 2055 \\
\hline 150 & 8 & 6 & 13.14 & 13.14 & 10.29 & 10.29 & 4.12 & 13.14 & 310.53 & 312.13 & 879.47 & 195.80 & 107.40 & 450.92 & 9465 & 3899 & 14650 & 903 & 457 & 4974 \\
\hline 150 & 10 & 6 & 8.34 & 8.34 & 5.63 & 5.63 & 5.55 & 8.34 & 545.08 & 269.56 & 348.89 & 157.98 & 653.42 & 208.04 & 5125 & 2907 & 773 & 438 & 1353 & 2306 \\
\hline \multicolumn{3}{|c|}{$\begin{array}{l}\text { Average } \\
\text { Avo }\end{array}$} & 16.71 & 16.71 & 16.19 & 16.19 & 1.26 & 5.06 & $\begin{array}{r}512.58 \\
(0.06)\end{array}$ & $\begin{array}{c}528.53 \\
(0.02)\end{array}$ & $\begin{array}{c}579.71 \\
(0.03)\end{array}$ & $\begin{array}{c}371.14 \\
(0.02)\end{array}$ & $\begin{array}{c}190.60 \\
(0.00)\end{array}$ & $\begin{array}{r}420.15 \\
(0.05)\end{array}$ & 10497 & 14525 & 10823 & 7669 & 2571 & 5806 \\
\hline \multicolumn{3}{|c|}{ \# of solved ins. (/24) } & & & & & & & 22 & 23 & 22 & 23 & 24 & 23 & & & & & & \\
\hline \multicolumn{3}{|c|}{ \# of best solutions (/24) } & 6 & 6 & 6 & 6 & 24 & 9 & & 3 & 2 & 2 & 9 & 8 & & & 3 & 6 & 16 & 4 \\
\hline
\end{tabular}


Table 9

Results for CoverageVLP and C-type instances.

\begin{tabular}{|c|c|c|c|c|c|c|c|c|c|c|c|c|c|c|c|c|c|c|c|c|}
\hline \multicolumn{3}{|c|}{ Parameters } & \multicolumn{6}{|c|}{ LP gap (\%) } & \multicolumn{6}{|c|}{ Cpu time (s)/optimality gap (\%) } & \multicolumn{6}{|c|}{ Number of nodes } \\
\hline$\rho_{\min }$ & $k^{\max }$ & $p$ & CMO & CM1 & CM2 & CM3 & CM4 & CM5 & CMO & $C M 1$ & CM2 & CM3 & CM4 & CM5 & CMO & $C M 1$ & CM2 & CM3 & CM4 & CM5 \\
\hline 50 & 6 & 4 & 25.73 & 25.73 & 25.71 & 25.71 & 1.19 & 1.19 & 432.22 & 900.92 & 3165.87 & 1843.91 & 150.42 & 158.94 & 25535 & 48016 & 165456 & 43576 & 5481 & 7935 \\
\hline 50 & 8 & 4 & 2.12 & 2.12 & 2.12 & 2.12 & 1.73 & 1.73 & 17.82 & 32.34 & 34.28 & 45.52 & 22.19 & 43.85 & 3541 & 8041 & 5304 & 5359 & 3325 & 7035 \\
\hline 50 & 10 & 4 & 0.00 & 0.00 & 0.00 & 0.00 & 0.00 & 0.00 & 0.45 & 0.50 & 0.81 & 0.94 & 0.91 & 0.46 & $\mathbf{0}$ & $\mathbf{0}$ & o & $\mathbf{0}$ & o & $\mathbf{0}$ \\
\hline 50 & 6 & 6 & 9.33 & 9.33 & 9.32 & 9.32 & 4.20 & 4.40 & 786.90 & 485.58 & 725.56 & 1299.77 & 533.40 & 261.94 & 43195 & 15868 & 14914 & 19261 & 5390 & 4445 \\
\hline 50 & 8 & 6 & 0.00 & 0.00 & 0.00 & 0.00 & 0.00 & 0.00 & 0.48 & 0.56 & 0.93 & 0.88 & 0.96 & 0.46 & $\mathbf{0}$ & $\mathbf{0}$ & o & $\mathbf{0}$ & $\mathbf{0}$ & $\mathbf{0}$ \\
\hline 50 & 10 & 6 & 0.00 & 0.00 & 0.00 & 0.00 & 0.00 & 0.00 & 0.53 & 0.67 & 0.90 & 0.96 & 0.73 & 0.67 & 0 & $\mathbf{0}$ & o & $\mathbf{0}$ & o & $\mathbf{0}$ \\
\hline 50 & 6 & 8 & 3.07 & 3.07 & 3.01 & 3.01 & 1.34 & 2.54 & 56.66 & 62.70 & 96.86 & 144.78 & 82.68 & 105.36 & 3140 & 3299 & 2386 & 2468 & 1164 & 5045 \\
\hline 50 & 8 & 8 & 0.04 & 0.04 & 0.02 & 0.02 & 0.02 & 0.04 & 2.06 & 1.45 & 0.64 & 0.79 & 0.82 & 1.38 & 163 & 40 & $\mathbf{0}$ & $\mathbf{0}$ & $\mathbf{0}$ & 20 \\
\hline 50 & 10 & 8 & 0.04 & 0.04 & 0.02 & 0.02 & 0.02 & 0.04 & 5.09 & 4.43 & 0.78 & 1.02 & 1.00 & 6.75 & 456 & 418 & $\mathbf{0}$ & $\mathbf{0}$ & o & 486 \\
\hline 100 & 6 & 4 & 25.73 & 25.73 & 25.71 & 25.71 & 1.19 & 1.19 & 342.98 & 379.13 & 477.49 & 1277.67 & 91.70 & 140.39 & 20271 & 19062 & 17330 & 30092 & 2723 & 7229 \\
\hline 100 & 8 & 4 & 2.12 & 2.12 & 2.12 & 2.12 & 1.73 & 1.73 & 85.01 & 25.16 & 14.24 & 29.73 & 15.64 & 92.02 & 11960 & 3464 & 1240 & 4053 & 1927 & 27160 \\
\hline 100 & 10 & 4 & 0.00 & 0.00 & 0.00 & 0.00 & 0.00 & 0.00 & 1.19 & 0.92 & 0.87 & 0.87 & 1.22 & 0.56 & $\mathbf{0}$ & 9 & $\mathbf{0}$ & $\mathbf{0}$ & $\mathbf{0}$ & $\mathbf{0}$ \\
\hline 100 & 6 & 6 & 9.33 & 9.33 & 9.32 & 9.32 & 4.20 & 4.40 & 284.11 & 541.96 & 388.66 & 1703.63 & 475.63 & 237.37 & 10200 & 13656 & 10011 & 23266 & 4724 & 4902 \\
\hline 100 & 8 & 6 & 0.00 & 0.00 & 0.00 & 0.00 & 0.00 & 0.00 & 0.42 & 1.56 & 1.02 & 1.02 & 0.81 & 0.53 & $\mathbf{0}$ & 57 & 0 & $\mathbf{0}$ & o & $\mathbf{0}$ \\
\hline 100 & 10 & 6 & 0.00 & 0.00 & 0.00 & 0.00 & 0.00 & 0.00 & 4.08 & 0.60 & 1.26 & 1.08 & 1.21 & 2.44 & 330 & $\mathbf{0}$ & 0 & $\mathbf{0}$ & $\mathbf{0}$ & 39 \\
\hline 100 & 6 & 8 & 3.56 & 3.56 & 3.13 & 3.13 & 1.76 & 3.09 & 196.36 & 273.46 & 191.69 & 180.66 & 192.90 & 170.01 & 8225 & 17602 & 4506 & 2313 & 1987 & 8702 \\
\hline 100 & 8 & 8 & 0.88 & 0.88 & 0.40 & 0.40 & 0.38 & 0.88 & 11.23 & 11.54 & 10.08 & 6.99 & 1.41 & 12.65 & 482 & 581 & 43 & 28 & $\mathbf{0}$ & 474 \\
\hline 100 & 10 & 8 & 0.88 & 0.88 & 0.40 & 0.40 & 0.40 & 0.88 & 13.58 & 18.82 & 25.41 & 17.09 & 4.97 & 14.22 & 533 & 709 & 415 & 170 & 12 & 525 \\
\hline 150 & 6 & 4 & 25.73 & 25.73 & 25.71 & 25.71 & 1.19 & 1.19 & 367.43 & 383.30 & 1142.58 & 2294.78 & 172.68 & 91.51 & 17763 & 18110 & 61517 & 64122 & 5660 & 4664 \\
\hline 150 & 8 & 4 & 2.12 & 2.12 & 2.12 & 2.12 & 1.73 & 1.73 & 73.07 & 38.82 & 12.76 & 61.09 & 79.50 & 11.40 & 7779 & 4756 & 1314 & 6003 & 9639 & 1095 \\
\hline 150 & 10 & 4 & 0.06 & 0.06 & 0.06 & 0.06 & 0.06 & 0.06 & 1.48 & 5.31 & 3.39 & 4.54 & 2.66 & 7.63 & 528 & 667 & 972 & 558 & 230 & 1002 \\
\hline 150 & 6 & 6 & 9.33 & 9.33 & 8.99 & 8.99 & 4.20 & 4.40 & 572.66 & 417.78 & 585.98 & 1927.39 & 473.40 & 267.09 & 15987 & 10033 & 10880 & 19465 & 4232 & 6283 \\
\hline 150 & 8 & 6 & 0.13 & 0.13 & 0.06 & 0.06 & 0.06 & 0.13 & 4.86 & 2.34 & 3.61 & 1.16 & 1.06 & 8.42 & 283 & 64 & 20 & $\mathbf{0}$ & $\mathbf{0}$ & 474 \\
\hline 150 & 10 & 6 & 0.13 & 0.13 & 0.06 & 0.06 & 0.06 & 0.13 & 5.03 & 6.50 & 1.32 & 1.36 & 1.56 & 5.78 & 457 & 402 & $\mathbf{0}$ & $\mathbf{0}$ & $\mathbf{0}$ & 332 \\
\hline \multicolumn{3}{|c|}{$\begin{array}{l}\text { Average } \\
\text { Avg. opt. gap (\%) }\end{array}$} & 5.02 & 5.02 & 4.93 & 4.93 & 1.06 & 1.24 & $\begin{array}{c}135.70 \\
(\mathbf{0 . 0 0})\end{array}$ & $\begin{array}{c}149.85 \\
(\mathbf{0 . 0 0})\end{array}$ & $\begin{array}{r}286.96 \\
(\mathbf{0 . 0 0})\end{array}$ & $\begin{array}{c}451.98 \\
(\mathbf{0 . 0 0})\end{array}$ & $\begin{array}{l}96.23 \\
(\mathbf{0 . 0 0})\end{array}$ & $\begin{array}{l}68.41 \\
(0.00)\end{array}$ & 7118 & 6869 & 12346 & 9197 & 1937 & 3660 \\
\hline \multicolumn{3}{|c|}{ \# of solved ins. (/24) } & & & & & & & 24 & 24 & 24 & 24 & 24 & 24 & & & & & & \\
\hline \multicolumn{3}{|c|}{ \# of best solutions (/24) } & 7 & 7 & 12 & 12 & 24 & 13 & 5 & 1 & 4 & & 6 & 8 & 5 & 3 & 9 & 10 & 20 & 8 \\
\hline
\end{tabular}


Table 10

Results for CoverageVLP and D-type instances.

\begin{tabular}{|c|c|c|c|c|c|c|c|c|c|c|c|c|c|c|c|c|c|c|c|c|}
\hline \multicolumn{3}{|c|}{ Parameters } & \multicolumn{6}{|c|}{ LP gap (\%) } & \multicolumn{6}{|c|}{ Cpu time (s)/optimality gap (\%) } & \multicolumn{6}{|c|}{ Number of nodes } \\
\hline$\rho_{\min }$ & $k^{\max }$ & $p$ & CMO & CM1 & CM2 & CM3 & CM4 & CM5 & CMO & $C M 1$ & CM2 & CM3 & $C M 4$ & CM5 & CMO & CM1 & CM2 & CM3 & CM4 & CM5 \\
\hline 50 & 6 & 4 & 25.73 & 25.73 & 25.71 & 25.71 & 1.19 & 1.19 & 353.54 & 609.85 & 598.64 & 758.12 & 259.94 & 92.84 & 17552 & 34889 & 17870 & 20915 & 7610 & 4460 \\
\hline 50 & 8 & 4 & 2.12 & 2.12 & 2.12 & 2.12 & 1.73 & 1.73 & 47.49 & 19.55 & 35.59 & 11.86 & 15.30 & 47.08 & 7082 & 2734 & 4079 & 1497 & 1449 & 5086 \\
\hline 50 & 10 & 4 & 0.00 & 0.00 & 0.00 & 0.00 & 0.00 & 0.00 & 0.41 & 0.42 & 0.97 & 0.92 & 0.99 & 0.52 & $\mathbf{0}$ & $\mathbf{0}$ & $\mathbf{0}$ & $\mathbf{0}$ & $\mathbf{0}$ & $\mathbf{0}$ \\
\hline 50 & 6 & 6 & 9.33 & 9.33 & 9.32 & 9.32 & 4.20 & 4.40 & 565.19 & 349.39 & 510.66 & 2235.29 & 490.20 & 669.14 & 15258 & 10412 & 18273 & 36960 & 5097 & 8462 \\
\hline 50 & 8 & 6 & 0.00 & 0.00 & 0.00 & 0.00 & 0.00 & 0.00 & 1.16 & 0.51 & 0.90 & 1.09 & 0.91 & 1.30 & 70 & $\mathbf{0}$ & $\mathbf{0}$ & $\mathbf{0}$ & $\mathbf{0}$ & $\mathbf{0}$ \\
\hline 50 & 10 & 6 & 0.00 & 0.00 & 0.00 & 0.00 & 0.00 & 0.00 & 2.04 & 1.88 & 0.98 & 1.11 & 1.20 & 1.25 & 69 & 51 & o & $\mathbf{0}$ & $\mathbf{0}$ & 39 \\
\hline 50 & 6 & 8 & 3.20 & 3.20 & 3.12 & 3.12 & 1.46 & 2.67 & 48.02 & 36.32 & 113.23 & 113.57 & 104.06 & 119.09 & 3000 & 1902 & 3122 & 1808 & 1366 & 6319 \\
\hline 50 & 8 & 8 & 0.04 & 0.04 & 0.02 & 0.02 & 0.02 & 0.04 & 2.39 & 2.92 & 1.06 & 0.86 & 0.70 & 3.69 & 156 & 270 & $\mathbf{0}$ & $\mathbf{0}$ & $\mathbf{0}$ & 342 \\
\hline 50 & 10 & 8 & 0.04 & 0.04 & 0.02 & 0.02 & 0.02 & 0.04 & 4.06 & 3.19 & 0.86 & 0.86 & 0.80 & 5.31 & 363 & 255 & $\mathbf{0}$ & $\mathbf{0}$ & $\mathbf{0}$ & 400 \\
\hline 100 & 6 & 4 & 25.73 & 25.73 & 25.71 & 25.71 & 1.19 & 1.19 & 334.94 & 430.85 & 779.60 & 2111.85 & 243.26 & 110.99 & 15638 & 23082 & 27070 & 47622 & 7012 & 4881 \\
\hline 100 & 8 & 4 & 2.12 & 2.12 & 2.12 & 2.12 & 1.73 & 1.73 & 22.64 & 36.73 & 17.40 & 38.84 & 107.88 & 45.60 & 6179 & 5577 & 1087 & 4738 & 11227 & 6980 \\
\hline 100 & 10 & 4 & 0.00 & 0.00 & 0.00 & 0.00 & 0.00 & 0.00 & 0.56 & 0.48 & 0.87 & 0.86 & 0.88 & 0.54 & o & o & $\mathbf{0}$ & o & 0 & $\mathbf{0}$ \\
\hline 100 & 6 & 6 & 9.33 & 9.33 & 9.32 & 9.32 & 4.20 & 4.40 & 269.34 & 637.17 & 633.62 & 1841.72 & 468.53 & 186.58 & 9873 & 14758 & 10579 & 26504 & 5138 & 3494 \\
\hline 100 & 8 & 6 & 0.00 & 0.00 & 0.00 & 0.00 & 0.00 & 0.00 & 3.58 & 1.58 & 1.17 & 1.60 & 1.19 & 2.98 & 365 & 46 & $\mathbf{0}$ & $\mathbf{0}$ & o & 474 \\
\hline 100 & 10 & 6 & 0.00 & 0.00 & 0.00 & 0.00 & 0.00 & 0.00 & 5.05 & 5.59 & 5.35 & 1.24 & 1.32 & 2.94 & 486 & 500 & 474 & $\mathbf{0}$ & $\mathbf{0}$ & 61 \\
\hline 100 & 6 & 8 & 3.68 & 3.68 & 2.99 & 2.99 & 1.82 & 1.82 & 420.18 & 321.45 & 206.31 & 415.81 & 311.55 & 395.33 & 35763 & 16979 & 8808 & 4794 & 3219 & 18690 \\
\hline 100 & 8 & 8 & 1.21 & 1.21 & 0.64 & 0.64 & 0.64 & 0.68 & $(0.23)$ & $(0.05)$ & $(0.28)$ & 41.16 & 86.22 & $(0.31)$ & 104629 & 69792 & 94567 & 515 & 952 & 97678 \\
\hline 100 & 10 & 8 & 1.21 & 1.21 & 0.64 & 0.64 & 0.64 & 1.21 & $(0.32)$ & $(0.31)$ & $(0.29)$ & 123.07 & 85.88 & $(0.32)$ & 117575 & 65763 & 60112 & 1633 & 1493 & 118411 \\
\hline 150 & 6 & 4 & 25.73 & 25.73 & 25.71 & 25.71 & 1.19 & 1.19 & 1801.76 & 345.84 & 447.40 & 1900.28 & 187.28 & 105.06 & 94078 & 17714 & 18728 & 54512 & 4178 & 5010 \\
\hline 150 & 8 & 4 & 2.12 & 2.12 & 2.12 & 2.12 & 1.73 & 1.73 & 14.85 & 60.46 & 40.80 & 13.44 & 39.14 & 70.16 & 1580 & 5394 & 6144 & 1782 & 4801 & 8268 \\
\hline 150 & 10 & 4 & 0.06 & 0.06 & 0.06 & 0.06 & 0.06 & 0.06 & 4.72 & 6.56 & 5.40 & 2.53 & 3.87 & 6.35 & 827 & 559 & 569 & 161 & 254 & 760 \\
\hline 150 & 6 & 6 & 9.33 & 9.33 & 8.98 & 8.98 & 4.20 & 4.40 & 431.83 & 363.12 & 985.60 & 1289.40 & 697.38 & 187.72 & 10803 & 8963 & 23610 & 14116 & 5708 & 3302 \\
\hline 150 & 8 & 6 & 0.13 & 0.13 & 0.04 & 0.04 & 0.04 & 0.13 & 2.34 & 1.72 & 2.53 & 0.92 & 1.04 & 7.82 & 141 & 50 & 20 & $\mathbf{0}$ & o & 240 \\
\hline 150 & 10 & 6 & 0.13 & 0.13 & 0.04 & 0.04 & 0.04 & 0.13 & 0.72 & 1.60 & 1.34 & 1.46 & 1.37 & 0.81 & $\mathbf{0}$ & 23 & $\mathbf{0}$ & $\mathbf{0}$ & $\mathbf{0}$ & $\mathbf{0}$ \\
\hline \multicolumn{3}{|c|}{$\begin{array}{l}\text { Average } \\
\text { Avo }\end{array}$} & 5.03 & 5.03 & 4.94 & 4.94 & 1.09 & 1.20 & $\begin{array}{c}480.71 \\
(0.02)\end{array}$ & $\begin{array}{r}434.89 \\
(0.01)\end{array}$ & $\begin{array}{c}482.94 \\
(0.02)\end{array}$ & $\begin{array}{c}454.49 \\
(\mathbf{0 . 0 0})\end{array}$ & $\begin{array}{c}129.62 \\
(0.00)\end{array}$ & $\begin{array}{c}385.98 \\
(\mathbf{0 . 0 3})\end{array}$ & 18395 & 11655 & 12296 & 9065 & 2479 & 12223 \\
\hline \multicolumn{3}{|c|}{ \# of solved ins. (/24) } & & & & & & & 22 & 22 & 22 & 24 & 24 & 22 & & & & & & \\
\hline \multicolumn{3}{|c|}{ \# of best solutions (/24) } & 7 & 7 & 13 & 13 & 24 & 14 & 2 & 4 & 4 & 6 & 3 & 5 & 4 & 3 & 8 & 12 & 15 & 7 \\
\hline
\end{tabular}


$\rho_{\min }=100, k_{j}^{\max }=8$, and $p=6$ for $\mathbf{A}$ - and D-types. The solutions are depicted in Figs. 2-5. In all these figures, the locations of vendors are denoted by rectangles and their service regions are marked by different colors. Demand points that are not served by any of the vendors are not colored. The areas that are not population zones are not numbered.

Table 11

Improvements in linear programming bounds.

\begin{tabular}{llllllll}
\hline \multirow{2}{*}{ Type } & \multicolumn{2}{l}{ ProfitVLP } & & \multicolumn{2}{l}{ Coverage VLP } \\
\cline { 2 - 3 } & $(16)$ & $(18),(19),(25)-(27)$ & $(31)$ & & $(16)$ & $(18),(19),(25)-(27)$ & $(31)$ \\
\hline A & 0 & 30.23 & 9.77 & 0 & 0.33 & 8.24 \\
B & 0 & 30.65 & 9.64 & 0 & 0.43 & 8.24 \\
C & 0 & 3.22 & 3.80 & 0 & 0.09 & 3.09 \\
D & 0 & 3.31 & 3.75 & 0 & 0.10 & 3.09 \\
\hline
\end{tabular}

An optimal solution for ProfitVLP for an A-type instance is given in Fig. 2. Here we can see that as the demands and profits of demand points are independent of the distances to the vendors, the service regions are quite dispersed. For instance, demand point 14 , which is assigned to the vendor at location 7 , is surrounded by three other demand points that are all served by the vendor at location 15 . Similarly, demand point 57 is served by the vendor at location 65 even though there is another vendor at a neighboring location.

We see an optimal solution for ProfitVLP for a D-type instance in Fig. 3. Here we observe that the service regions of vendors are rather compact and the vendors are located more centrally in their regions.

Optimal solutions for CoverageVLP for A- and D-type instances are given in Figs. 4 and 5, respectively. We see a similar pattern here, i.e., the service regions are more compact in the solution for the D-type instance.

Table 12

Best profit and coverage values.

\begin{tabular}{|c|c|c|c|c|c|c|c|c|c|c|}
\hline \multicolumn{3}{|c|}{ Parameters } & \multicolumn{4}{|c|}{ ProfitVLP } & \multicolumn{4}{|c|}{ Coverage VLP } \\
\hline$\rho_{\min }$ & $k^{\max }$ & $p$ & A-type & B-type & C-type & D-type & A-type & B-type & C-type & D-type \\
\hline 50 & 6 & 4 & 832.8 & 806.1 & 852.8 & 828.8 & 1413 & 1413 & 1423 & 1423 \\
\hline 50 & 8 & 4 & 1006.8 & 974.2 & 1033.8 & 1005.0 & 1790 & 1790 & 1761 & 1761 \\
\hline 50 & 10 & 4 & 1053.3 & 1016.9 & 1033.8 & 1005.0 & 2095 & 2095 & 1799 & 1799 \\
\hline 50 & 6 & 6 & 1094.2 & 1057.7 & 1158.7 & 1128.0 & 2042 & 2042 & 2069 & 2069 \\
\hline 50 & 8 & 6 & 1152.7 & 1113.2 & 1250.7 & 1214.3 & 2450 & 2450 & 2263 & 2263 \\
\hline 50 & 10 & 6 & 1160.2 & 1119.6 & 1250.7 & 1214.3 & 2677 & 2677 & 2263 & 2263 \\
\hline 50 & 6 & 8 & 1070.6 & 1031.5 & 1250.7 & 1197.9 & 2508 & 2508 & 2435 & 2432 \\
\hline 50 & 8 & 8 & 1071.6 & 1035.3 & 1271.1 & 1236.4 & 2739 & 2739 & 2509 & 2509 \\
\hline 50 & 10 & 8 & 1071.6 & 1035.3 & 1270.6 & 1236.2 & 2739 & 2739 & 2509 & 2509 \\
\hline 100 & 6 & 4 & 832.8 & 806.1 & 852.8 & 828.8 & 1413 & 1413 & 1423 & 1423 \\
\hline 100 & 8 & 4 & 1006.8 & 974.2 & 1033.8 & 1005.0 & 1790 & 1790 & 1761 & 1761 \\
\hline 100 & 10 & 4 & 1053.3 & 1016.9 & 1033.8 & 1005.0 & 2095 & 2095 & 1799 & 1799 \\
\hline 100 & 6 & 6 & 1094.2 & 1057.7 & 1158.7 & 1128.0 & 2042 & 2042 & 2069 & 2069 \\
\hline 100 & 8 & 6 & 1152.7 & 1112.8 & 1250.7 & 1214.3 & 2446 & 2446 & 2263 & 2263 \\
\hline 100 & 10 & 6 & 1160.2 & 1119.2 & 1250.7 & 1214.3 & 2677 & 2677 & 2263 & 2263 \\
\hline 100 & 6 & 8 & 1070.6 & 1024.8 & 1231.6 & 1197.9 & 2495 & 2495 & 2422 & 2420 \\
\hline 100 & 8 & 8 & 1070.6 & 1030.2 & 1271.1 & 1236.4 & 2739 & 2739 & 2488 & 2480 \\
\hline 100 & 10 & 8 & 1070.6 & 1031.6 & 1270.6 & 1236.4 & 2739 & 2739 & 2488 & 2480 \\
\hline 150 & 6 & 4 & 832.8 & 806.1 & 852.8 & 828.8 & 1413 & 1413 & 1423 & 1423 \\
\hline 150 & 8 & 4 & 1006.8 & 974.2 & 1033.8 & 1005.0 & 1790 & 1790 & 1761 & 1761 \\
\hline 150 & 10 & 4 & 1053.3 & 1016.9 & 1033.8 & 1005.0 & 2095 & 2095 & 1798 & 1798 \\
\hline 150 & 6 & 6 & 1074.7 & 1042.4 & 1158.7 & 1122.0 & 2032 & 2032 & 2069 & 2069 \\
\hline 150 & 8 & 6 & 1116.7 & 1079.3 & 1234.7 & 1198.3 & 2382 & 2366 & 2260 & 2260 \\
\hline 150 & 10 & 6 & 1124.2 & 1085.9 & 1234.7 & 1198.3 & 2502 & 2471 & 2260 & 2260 \\
\hline
\end{tabular}

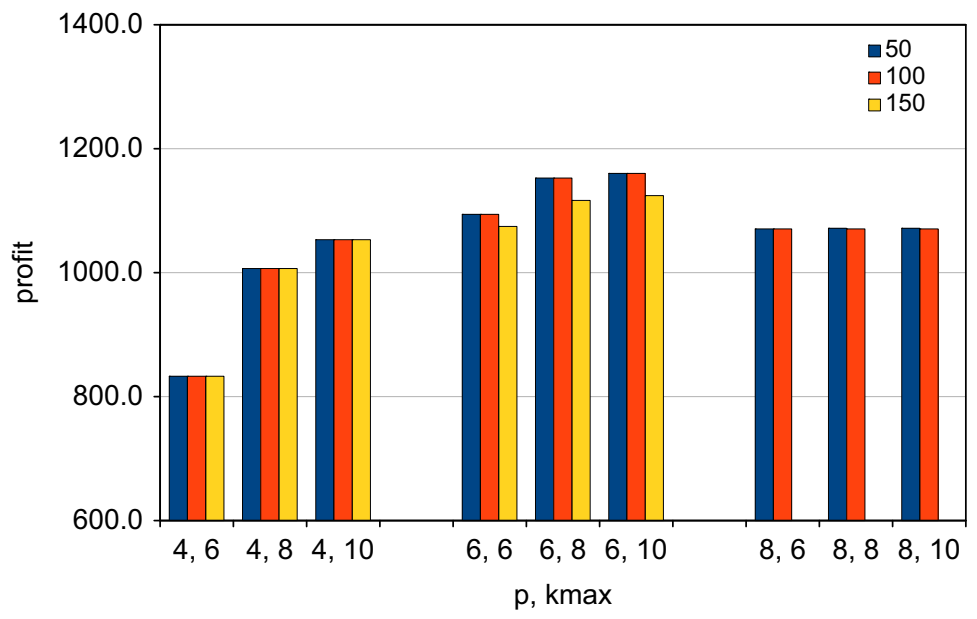

Fig. 1. Best profit values for A-type instances. 


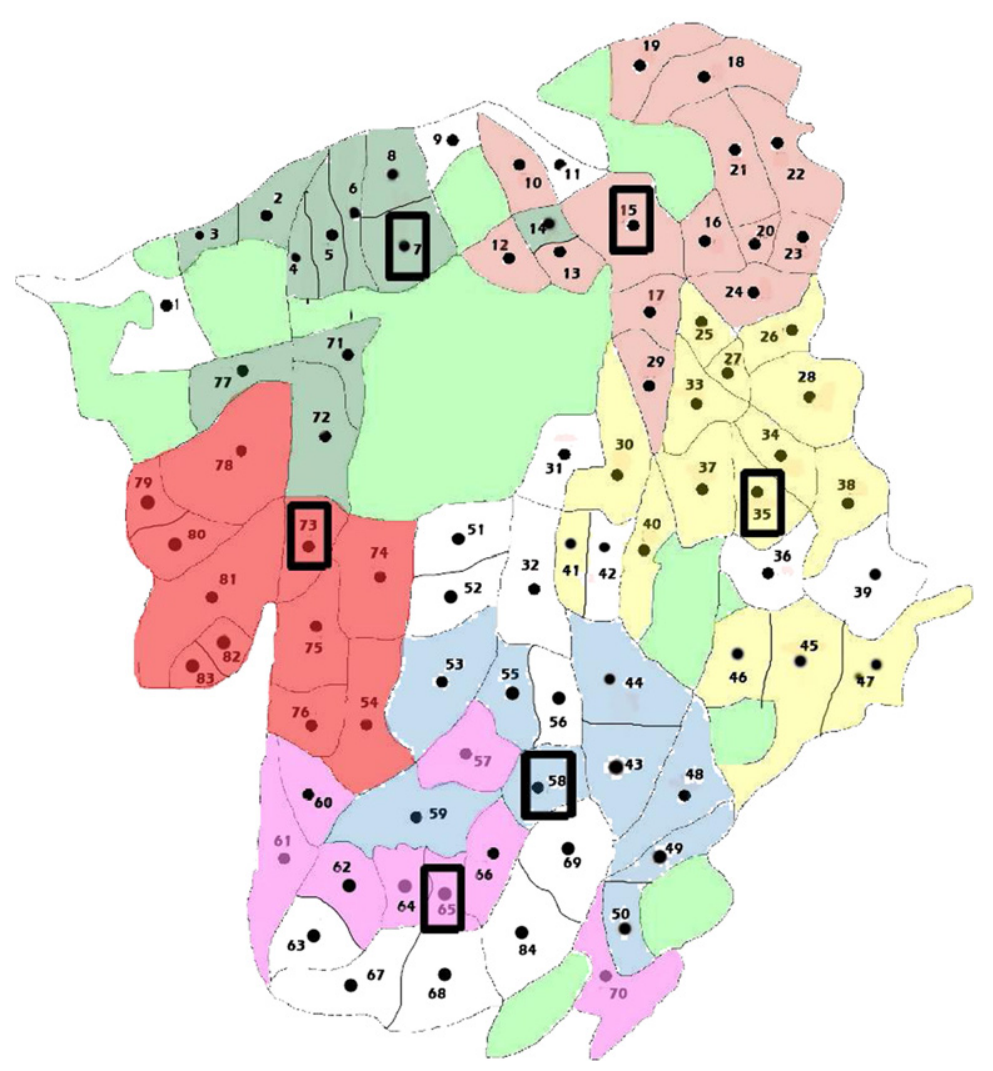

Fig. 2. Optimal solution of ProfitVLP for an A-type instance.

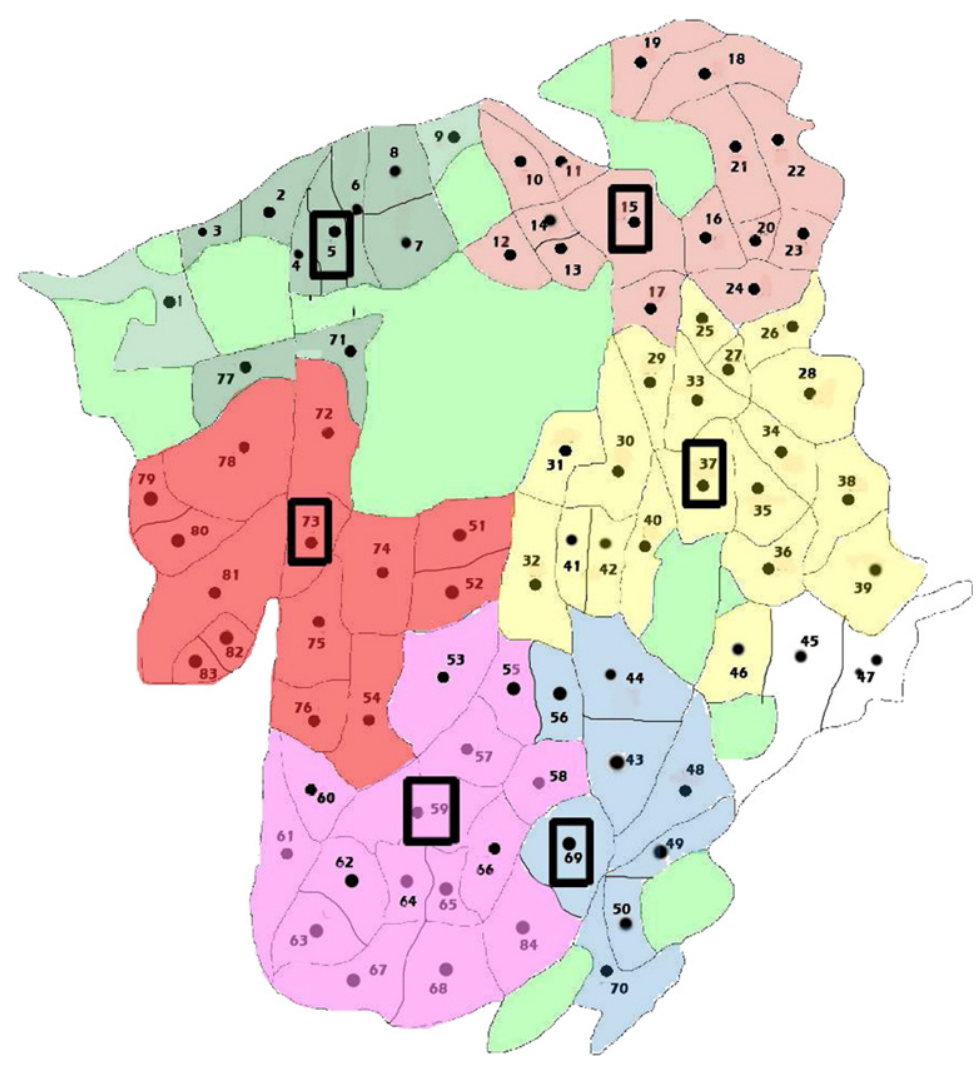

Fig. 3. Optimal solution of ProfitVLP for a D-type instance.

In summary, comparing these solutions, we see that demand points assigned to the same vendor lie around the vendor node for both ProfitVLP and CoverageVLP type D problems, whereas some demand points serviced from the same vendor are separated from the group in ProfitVLP and CoverageVLP for type A problems. This is expected since in A-type problems, profits and demands do not 


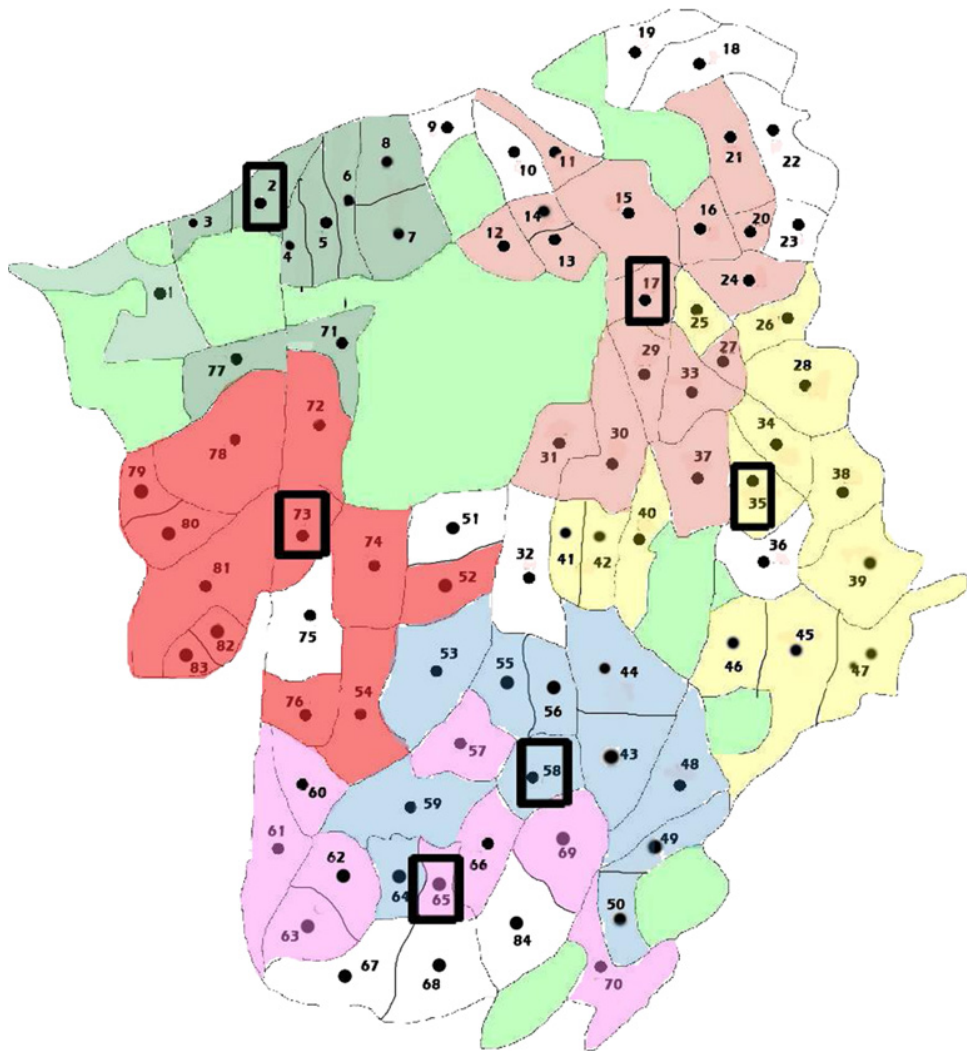

Fig. 4. Optimal solution of CoverageVLP for an A-type instance.

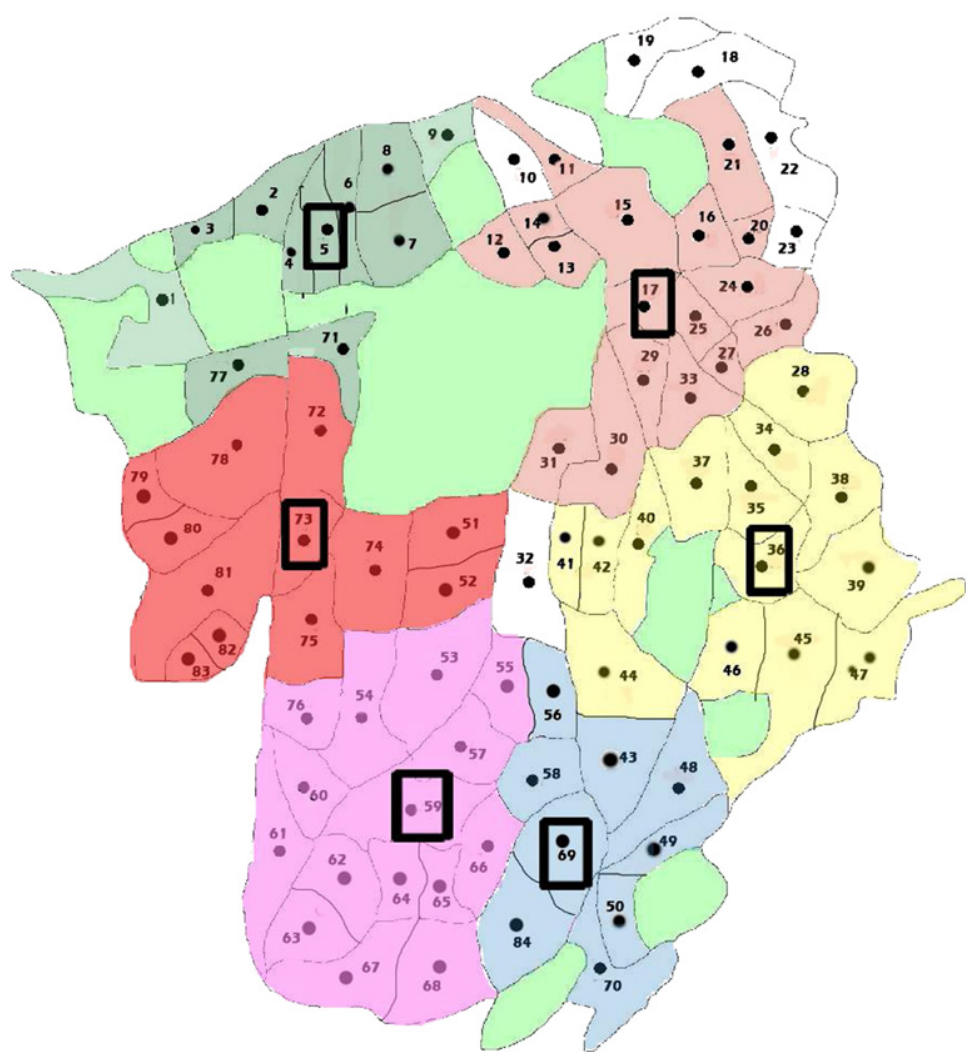

Fig. 5. Optimal solution of CoverageVLP for a D-type instance. 
depend on the distances between demand points and their vendors.

Moreover, the number of demand points served is larger in type D problems compared to type A problems. This is again expected as the profits and demands decrease as distances increase in D-type instances.

The total profits are $1152.70,1214.32,1032.20$, and 992.36 and the amounts of demand covered are 2180, 2229, 2446, and 2263 for ProfitVLP for type A, ProfitVLP for type D, CoverageVLP for type $\mathbf{A}$, and CoverageVLP for type $\mathbf{D}$ instances, respectively.

\section{Conclusion}

In this study, motivated by a real life application, we introduced the vendor location problem. We considered two versions of the problem with different objective functions. We proved that both versions of the problem are strongly NP-hard and suggested valid inequalities to strengthen the integer programming formulations and to reduce the solution times.

Our computational experiments showed that the bounds of the linear programming relaxations of the problem with profit maximization objective are quite poor in quality and it is very difficult to solve these problems to optimality with integer programming solvers. Our valid inequalities strengthened our formulations significantly and reduced the computation times, however their effect was highly dependent on the instance. We also observed that the problem with the coverage objective was relatively easier to solve and valid inequalities were also useful in reducing the solution times for the instances of this problem.

We solved instances with different demand and profit functions and observed that the problems with profit maximization objective, where the demands change as a function of the distances between the demand points and their vendors are more difficult to solve compared to others. For some of these instances, we could not reach an optimal solution with any of our models. Even though the final gaps are not very large, still, we believe that alternative methods can be developed for these kinds of problems.

\section{Acknowledgment}

This research is supported by TUBITAK Project no. 107M460.

\section{References}

[1] Aardal K, Chudak FA, Shmoys DB. A 3-approximation algorithm for the k-level uncapacitated facility location problem. Information Processing Letters 1999;72:161-7.

[2] Aardal K, Labbé M, Leung J, Queyranne M. On the two-level uncapacitated facility location problem. INFORMS Journal on Computing 1996;8:289-301.

[3] Ageev A. Improved approximation algorithms for multilevel facility location problems. Operations Research Letters 2002;30:327-32.

[4] Ageev A, Ye Y, Zhang J. Improved combinatorial approximation algorithms for the k-level facility location problem. SIAM Journal on Discrete Mathematics 2004;18:207-17.

[5] Albareda-Sambola M, Fernández E, Laporte G. The capacity and distance constrained plant location problem. Computers \& Operations Research 2009;36:597-611.
[6] Barcelo J, Casanovas J. A heuristic Lagrangian algorithm for the capacitated plant location problem. European Journal of Operational Research 1984;15: 212-26.

[7] Barros AI, Labbé M. A general model for the uncapacitated facility and depot location problem. Location Science 1994;2:173-91.

[8] Bloemhof-Ruwaard JM, Salomon M, van Wassenhove LN. On the coordination of product and by-product flows in two-level distribution networks: model formulations and solution procedures. European Journal of Operational Research 1994;79:325-39.

[9] Bumb A. An approximation algorithm for the maximization version of the two level uncapacitated facility location problem. Operations Research Letters 2001;29:155-61.

[10] Bumb A, Kern W. A simple dual ascent algorithm for the multilevel facility location problem. Lecture Notes in Computer Science 2001;2129:55-63.

[11] Chardaire P, Lutton J-L, Sutter A. Upper and lower bounds for the two-level simple plant location problem. Annals of Operations Research 1999;86: 117-40.

[12] Gao JJ, Robinson Jr. EP. A dual-based optimization procedure for the twoechelon uncapacitated facility location problem. Naval Research Logistics 1992;839:191-212.

[13] Gao JJ, Robinson Jr. EP. Uncapacitated facility location: general solution procedure and computational experience. European Journal of Operational Research 1994;76:410-27.

[14] Gabor AF, van Ommeren JKCW. A new approximation algorithm for the multilevel facility location problem. Discrete Applied Mathematics 2010; 158:453-60.

[15] Garey MR, Johnson DS. Computers and intractability, a guide to the theory of NP-completeness. New York: Freeman; 1979.

[16] Guha S, Meyerson A, Munagala K. Hierarchical placement and network design problems. In: Proceedings of the 41st annual IEEE symposium on foundations of computer science; 2000. p. 603612.

[17] Holmberg K, Rönnqvist M, Yuan D. An exact algorithm for the capacitated facility location problems with single sourcing. European Journal of Operational Research 1999;113:544-59.

[18] Kaufman L, Vanden Eede M, Hansen P. A plant and warehouse location problem. Operational Research Quarterly 1997;28:547-57.

[19] Klincewicz J, Luss H. A Lagrangian relaxation heuristic for capacitated facility location with single-source constraints. Journal of the Operational Research Society $1986 ; 37: 495-500$.

[20] Marín A. Lower bounds for the two-stage uncapacitated facility location problem. European Journal of Operational Research 2007;179:1126-42.

[21] Marín A, Pelegrín B. Applying Lagrangean relaxation to the resolution of twostage location problems. Annals of Operations Research 1999;86:179-98.

[22] Meyerson A, Munagala K, Plotkin S. Cost-distance: two-metric network design. In: Proceedings of the 41st IEEE symposium on foundations of computer science, FOCS; 2000. p. 624-30.

[23] Neebe A, Rao M. An algorithm for the fixed-charge assigning users to sources problem. Journal of the Operational Research Society 1983;34:1107-13.

[24] Pirkul H, Jayaraman V. Production, transportation, and distribution planning in a multi-commodity tri-echelon system. Transportation Science 1996;30: 291-302.

[25] Ro H, Tcha D. A branch-and-bound algorithm for the two-level uncapacitated facility location problem with some side constraints. European Journal of Operational Research 1984;18:349-58.

[26] Shmoys DB, Tardos E, Aardal K. Approximation algorithms for facility location problems. In: Proceedings of the 29th annual ACM symposium on theory of computing; 1997. p. 265-74.

[27] SUDER 〈http://www.suder.org.tr/sektor.html〉; 2009.

[28] Şahin G, Süral H. A review of hierarchical location models. Computers \& Operations Research 2007;34:2310-31.

[29] Tcha D, Lee B. A branch-and-bound algorithm for the multi-level uncapacitated facility location problem. European Journal of Operational Research 1984;18:35-43.

[30] Tragantalerngsak S, Holt J, Ronnqvist M. Lagrangian relaxation heuristic for two-echelon single-source capacitated facility location problem. European Journal of Operational Research 1997;102:611-25.

[31] Tragantalerngsak S, Holt J, Ronnqvist M. An exact method for the twoechelon, single-source, capacitated facility location problem. European Journal of Operational Research 2000;123:473-89.

[32] Zhang J. Approximating the two-level facility location problem via a quasigreedy approach. Mathematical Programming 2006;108:159-76.

[33] Zhang J, Ye Y. A note on the maximization version of the multi-level facility location problem. Operations Research Letters 2002;30:333-5. 